AIAA-2002-1261

\title{
DEPLOYMENT SIMULATION OF ULTRA-LIGHTWEIGHT INFLATABLE STRUCTURES
}

\author{
John T. Wang* and Arthur R. Johnson ${ }^{\dagger}$ \\ NASA Langley Research Center \\ Hampton, VA
}

\begin{abstract}
$\underline{\text { Abstract }}$
Dynamic deployment analyses of folded inflatable tubes are conducted to investigate modeling issues related to the deployment of solar sail booms. The analyses are necessary because ground tests include gravity effects and may poorly represent deployment in space. A control volume approach, available in the LS-DYNA nonlinear dynamic finite element code, and the ideal gas law are used to simulate the dynamic inflation deployment process. Three deployment issues are investigated for a tube packaged in a Z-fold configuration. The issues are the effect of the rate of inflation, the effect of residual air, and the effect of gravity. The results of the deployment analyses reveal that the time and amount of inflation gas required to achieve a full deployment are related to these issues.
\end{abstract}

\section{$\underline{\text { Introduction }}$}

Ultra-Lightweight Inflatable (ULI) space structures have become attractive because they can meet structural requirements for space applications at a low cost. These thin membrane structures can be fabricated and deployed for millions of dollars less than conventional structures. The roles envisioned for inflatable structures include solar sails, space telescope sunshades, solar arrays, pressurized habitats in space, antenna reflectors, and optical telescope mirrors. Solar sails capture the momentum of sunlight photons. The area density of the available momentum is small. As a result, solar sails must be large. The thrust provided by these large sails is so small that the sails must also be thinner than paper to produce useful thrust vectors on the spacecraft. Billowing solar sails are less effective than flat solar sails due to the reduced momentum exchange provided by oblique incidence photons. To keep billowing within allowable limits, membrane tension is provided. The tension can be introduced by employing inflatable

* Aerospace Engineer, AIAA senior member.

$\uparrow$ Mechanical Engineer, Army Research Laboratory.

Copyright (c) 2002 by the American Institute of Aeronautics and Astronautics, Inc. No copyright is asserted in the United States under Title 17, U.S. Code. The U.S. Government has a royalty-free license to exercise all rights under the copyright claimed herein for Governmental purposes. All other rights are reserved by the copyright owner. spars to stretch the membranes, by spinning the sail to produce a centrifugal force, or by a combination of these methods. Three solar sail configurations [1,2] under investigation recently are shown in Figure 1. They include a square solar sail configuration, a disc solar sail configuration, and a heliogyro solar sail configuration. The square solar sail uses inflatable spars for deployment and for maintaining the tension in the sail membranes, while the disc and heliogyro solar sails spin after deployment to generate centrifugal tension forces which help maintain the desired configuration. The disc solar sail also has inflatable spars to assist its deployment.

Developing and testing prototypes in space is prohibitively expensive. Deployment tests of solar sails in a laboratory do not accurately simulate their deployment in space. Even if a ground test is conducted in a vacuum chamber, the gravity effect can not be avoided. Gravity will affect the deployment dynamics, the structure's shape, and the regions experiencing selfcontact during the inflation deployment. Also, deployments that involve spinning the entire structure are very difficult to conduct in ground based laboratory tests. Thus, laboratory testing of ULI structures should be supplemented with computational simulations.

Many researchers have conducted computational simulations of the inflation deployment process for membrane structures. A closed form approximate analysis of the inflation deployment of a rolled (coiled) tube was derived by Steele and Fay [3]. The model gives insight into understanding the unrolling process. However, the unrolling tube is supported by an infinite plane. The constraint of the plane limits the application of this model for simulating deployment in-space. Haug et al [4] and Salama et al [5] employed control volume (CV) and finite element methods to investigate folded space rigidizable antenna structures and folded inflatable cylindrical tubes, respectively. The CV approach, implemented in the PAM-CRASH [6] and LS-DYNA [7] codes, is based on an airbag inflation model developed by Wang and Nefske [8]. As this approach neglects the inertia of the inflation gas, it may not be adequate for simulating deployment with a high velocity 
gas. To include the inflation gas inertia effect in the deployment simulation, the Arbitrary Lagrangian and Eulerian method (ALE) [9] needs to be used. However, the ALE method is computationally expensive since it requires the use of many Eulerian solid elements (for modeling the interior of the folded membrane structure.)

Dynamic deployment simulations often employ explicit integration algorithms that require small time steps. Depending on the complexity of the structure and on the total time required for the deployment, on the order of a million time steps may be needed to conduct an inflation deployment simulation. It is known that increasing the mass flow rate of the inflating gas shortens the deployment analysis time. However, it is still an open question as to whether the fast inflation analysis results can be extrapolated to predict the structural deployment behaviors at slow inflation rates.

Residual air, left inside the structure during packaging, can cause components to inflate simultaneously during the initial stages of deployment. In the NASA Inflatable Antenna Experiment (IAE) [10], unexpected deployment behavior was attributed to residual air. Thus, deployment simulations should include the effects of residual air.

The purpose of this study is to employ the CV approach, available in LS-DYNA, to simulate the dynamic deployment of two inflatable membrane structure configurations, and to investigate issues that are critical to the success of in-space inflation deployments. The issues investigated are the effects of inflation speed (mass flow rate), residual air, and gravity.

\section{Deployment Simulations}

\section{Inflation Modeling}

An inflatable membrane structure is modeled by discretizing it into a set of CVs. A longitudinal section of an inflatable tube and the inflation gas inside it form a $\mathrm{CV}$. The boundary of the CV, called the control surface, changes its shape as the internal pressure is increased. Very thin and soft membrane elements are placed between adjacent CVs. These membrane elements stretch as the CVs deform. The total area of the membrane elements are referred to as the orifice area which controls the flow of inflation gas between adjacent CVs. A brief outline of this method is presented below. A more detailed description of the inflation modeling including contact algorithm is available in the literature, $[7,8]$.
The incremental volume change for a $\mathrm{CV}$ depends on the net inflow-mass rate, the equation of state for the gas, and the dynamics of the membrane structure bounding the $\mathrm{CV}$. Assuming all variables are known at time $t-\Delta t$, an approximation of the internal energy, $E(t)$, in the $\mathrm{CV}$ at time $t$ is given by

$$
E(t)=E(t-\Delta t)+c_{p} \dot{m}(t) \Delta t T_{i n}
$$

where is $c_{p}$ is the specific heat at constant pressure, $\Delta t$ is the time step, $T_{i n}$ is the inflation gas temperature, and $\dot{m}(t)$ is the mass flow rate of the inflation gas.

The gas mass density, $\rho(t)$, within the $\mathrm{CV}$ is approximated from the mass flow rate as follows,

$$
\rho(t)=[m(t-\Delta t)+\dot{m}(t) \Delta t] / V(t-\Delta t)
$$

where $V(t-\Delta t)$ is the CVs volume at time $t-\Delta t$. According to the equation of state for an ideal gas, the pressure, $p(t)$, is calculated as,

$$
p(t)=(k-1) \rho(t) \frac{E(t)}{m(t)}
$$

where $k$ is the ratio of the specific heat at constant pressure to the specific heat at constant volume.

The pressure is used as input to the finite element analysis to determine the structural configuration at time $t$. The equation of motion of the inflatable structure has the form

$$
[M][\ddot{D}\}+[C]\{\dot{D}\}+[K]\{D\}=\left\{R^{\text {ext }}\right\}
$$

where $[M],[C]$, and $[K]$ are the global mass, damping, and stiffness matrices computed with respect to the current configuration, $\left\{R^{\text {ext }}\right\}$ is the external load vector; and $\{D\},\{\dot{D}\}$, and $\{\ddot{D}\}$ are displacement, velocity, and acceleration vectors with respect to the current configuration at time $t$. Employing an explicit approach, the finite difference form of Equation (4) is expressed as 


$$
\begin{aligned}
& {\left[\frac{1}{\Delta t^{2}}[M]+\frac{1}{2 \Delta t}[C]\right]\{D\}_{t}=} \\
& \left\{R^{e x t}\right\}_{t-\Delta t}-[K][D\}_{t-\Delta t} \\
& +\frac{1}{\Delta t^{2}}[M]\left(2\{D\}_{t-\Delta t}-\{D\}_{t-2 \Delta t}\right) \\
& +\frac{1}{2 \Delta t}[C]\{D\}_{t-2 \Delta t}
\end{aligned}
$$

where $\Delta t$ is the time step. Equation (5) is solved for $\{D\}_{t}$ and the structure's shape at time $t$ is then available. This method is very efficient when the damping is zero and the mass matrix is made to be diagonal by employing lumping procedures. Time steps on the order of $10^{-6}$ seconds are typically required for the deployment models. As a result, the "wall clock" time, for a desktop workstation, can be on the order of weeks to simulate the deployment of a structure which in real time inflates in a few minutes.

The work performed due to the volume expansion reduces the internal energy, therefore a modified internal energy, $E(t)^{*}$, can be obtained according to the internal energy evolution equation,

$$
E(t)^{*}=E(t)\left[\frac{V(t)}{V(t-\Delta t)}\right]^{1-k}
$$

where $V(t)$ is the volume at time $t$ computed using the divergence theorem.

\section{$\underline{\text { Baseline Studies }}$}

The inflation deployment of a straight tube from its packaged configuration to its inflated shape is studied below. Two packaged configurations are modeled. In the first model the tube is packaged in a coiled shape, and in the second model it is in a Z-fold shape. We define the tube as deployed when it appears to be fully extended and is free of kinks. Both models employ fully integrated Belytschko-Tsay 4-node membrane elements [11]. A number of simulations were made to observe the effects of the tube's finite element discretization on the opening dynamics. The studies indicated that the meshes employed below are refined enough to capture the physical differences occurring during deployment due to the rate of inflation, residual air, and gravity. Deployment simulations are made to address the effects of the rate of inflation, residual air, and gravity.

\section{$\underline{\text { Material Properties }}$}

Both the coiled and Z-folded models are polyethylene tubes with a diameter of 3.82 inches and a thickness of 0.006 inches. The values of the tube's Young's modulus, Poisson's ratio, and density used are 25,000 $\mathrm{psi}, 0.25$, and $0.033 \mathrm{lbm} / \mathrm{in}^{3}$, respectively. The inflation is air at $70^{\circ} \mathrm{F}$ with a molecular weight of 28.97 $1 \mathrm{bm} / \mathrm{lbmole}$. The gas flows in at one end of the tube with an inflation mass flow rate $(d m / d t)$ of $0.1 \times t$ $\mathrm{lbm} / \mathrm{sec}$.

\section{Coiled Tube Model}

The finite element model for the coiled tube packaged configuration is displayed in Figure 2. It consists of seven CVs and is created by employing a simple Archimedean spiral equation, $r=a \theta+r_{0}$. The symbol $a$ represents a constant, $\theta$ is the sweep angle, and $r_{0}$ is the initial radius as shown in Figure 2. The nodes are created starting at $\theta=0$ and $r=r_{0}$ and continue up to a user defined length. The unwinding of the coiled tube is shown in Figure 3. The volume and pressure as a function of time for each $\mathrm{CV}$ are shown in Figures 4 and 5 , respectively. The tube is fully deployed at time 0.35 sec. The pressure is $8 \mathrm{psi}$, which induces a hoop strain of $10 \%$.

\section{$\underline{\text { Z-Folded Tube Model }}$}

The finite element model for the Z-folded tube packaged configuration is displayed in Figure 6. Inflated shapes of the Z-folded tube in various stages of deployment are shown in Figure 7. The Z-folded model contains four CVs with three orifices that are placed at the three fold lines. The time required to fully deploy the tube is 0.19 sec. The volume and pressure as a function of time for each $\mathrm{CV}$ are shown in Figures 8 and 9, respectively. The nominal volume of a fully opened central CV is 91 $\mathrm{in}^{3}$. The pressure at time $0.19 \mathrm{sec}$ is $2.4 \mathrm{psi}$ and it induced a hoop strain of $3.0 \%$.

\section{Investigation of Deployment Issues Using Z-Folded Tube Model}

\section{Effect of Inflation Rate}

Inflation mass flow rates $(d m / d t)$ of $0.01 \times t \mathrm{lbm} / \mathrm{sec}$, $0.1 \times t \mathrm{lbm} / \mathrm{sec}$, and $1.0 \times t \mathrm{lbm} / \mathrm{sec}$ representing a slow, medium, and fast inflation are employed. Note, the medium inflation rate case is the baseline problem described above (see Figures 6 to 9.) 
The slow inflation deployment sequence is displayed in Figure 10. It is difficult to fully deploy the tube with a slow inflation rate. The tube reaches a shape at $0.4 \mathrm{sec}$ in which the last folded section will not swing upward until $1.38 \mathrm{sec}$. The sudden swing of the last section to obtain the inflated shape results in an abrupt volume increase of the last $\mathrm{CV}$, see Figure 11. At that moment the pressure is about 10 psi (see Figure 12), which is much higher than the medium inflation rate case. The simulation indicates that the slow inflation process does not induce enough kinetic energy to flip the last section upward at an early time. This is similar to a folded garden hose that can not straighten itself by the internal water pressure.

The results of the fast inflation rate simulation are displayed in Figures 13 to 15 . As expected the fast inflation rate results in a quick deployment, full deployment achieved at $0.085 \mathrm{sec}$. The medium inflation rate requires $0.19 \mathrm{sec}$ to deploy. Each section inflates sequentially for the fast inflation case as shown in Figures 13 and 14. The pressure buildups in the first and second CVs are higher than the buildups in the other two control volumes, see Figure 15.

The orifices located at the connections between the control volumes control the amount of inflation gas going into each control volume per unit time. Two additional $Z$-fold deployment models are created by putting the orifices at different locations. In the first model, a five $\mathrm{CV}$ model is created by removing the orifices at the fold lines and installing orifices at the midsection of each of the four legs of the $\mathrm{Z}$-folded model. In the second model, eight $\mathrm{CVs}$ are created by installing orifices at the three fold lines and at the midsections of each of the four legs. Deployments were simulated for each model using the three inflation rates mentioned above. The computed dynamic deployment characteristics were similar to the four $\mathrm{CV}$ Z-folded models discussed above.

The required inflation time, the total mass of the inflation gas, and the pressures in the first and last CVs at the time of full deployment are listed in Table 1 . In general, the deployment time is proportional to the inflation rate. The internal pressures at the moment of full deployment are nearly uniform for the slow and medium inflation rate cases (as indicated by the small pressure difference between the first $\mathrm{CV}$ and the last CV.) In the case of a fast inflation rate, the pressure difference between the first $\mathrm{CV}$ and the last control volume is large. Note, deployment with a low inflation rate requires a high inflation pressure and a large amount of inflation gas (to flip the last section of the Z-folded tube upward.) A fast inflation rate deployment also requires more inflation mass than the medium inflation rate deployment. It appears that the pressure distribution and the amount of inflation gas in the tube at the moment of full deployment can not be simply related to the inflation gas flow rate.

\section{$\underline{\text { Effect of Residual Air }}$}

The effect of the presence of residual air on the deployment of Z-folded tube is investigated. Each CV, after the first one, is assumed to have a residual air mass of $2.703 \times 10^{-5} \mathrm{lbm}$, which induces a pressure of $0.1 \mathrm{psi}$ for a fully opened CV. The first CV is inflated by the inlet gas only and does not have any residual air present. At the start of the deployment simulation, the residual air expands each subsequent $\mathrm{CV}$ causing them to push each other apart. These initial motions allow the inflation gas to flow between CVs easier, and allow for a low pressure deployment.

The results of the residual air deployment simulation at the slow inflation rate, $0.01 \times t \mathrm{lbm} / \mathrm{sec}$, are shown in Figures 16 to 18 . Again, the residual air causes the control volumes to expand simultaneously at an early time and push each other upward, see Figures 16 and 17. The residual air changed the deployment process to prevent kinking between the last two CVs and the tube reached full deployment at $0.470 \mathrm{sec}$, which is much faster than the slow inflation rate case studied above. The pressure of 1.5 psi at full deployment, see Figure 18 , is also much lower than the slow inflation rate case (pressure about 10 psi shown in Figure 12) obtained without residual air.

\section{Effect of Gravity}

Gravity adds a body force to the equation of motion. This additional force is expected to affect the accelerations, deformations and the contact between adjacent control volumes during inflation. Thus, the deployment process is expected to change. The gravity effect needs to be investigated before results from ground tests can be used to help predict deployment in space.

The gravity effect is studied using the Z-fold model described above. The model shown in Figure 6 is modified by adding two $1.5 \mathrm{lb}$ masses to the top end corner nodes of the tube (Nodes A and B). The masses are added to simulate an inertial resistance and gravity load similar to that expected when a complex structure is being deployed. The boundary conditions shown in Figure 6 are used again. A measure of the effect of gravity is the difference between the time to full 
deployment with gravity enforced and the time for the case without gravity enforced.

The results for the simulation without gravity enforced are displayed in Figures 19 to 21, and for the case with gravity enforced in Figures 22 to 24 . Comparing Figures 19 and 22 a longer deployment time is required for the deployment with gravity, $0.60 \mathrm{sec}$ versus 0.485 sec. The intermediate deployment shapes are also quite different (see Figures 19 and 22). These differences can be observed by reviewing the expansion history of each CV. Comparing Figures 20 and 23, the third CV inflates faster in the presence of gravity. Also, a higher deployment pressure is required to overcome the additional forces due to gravity (compare Figures 21 and 24).

\section{$\underline{\text { Concluding Remarks }}$}

The control volume (CV) approach, as implemented in LS-DYNA code, was employed to simulate the inflation deployment of a tube when packaged first in a coiled tube configuration and second, when packaged in a Zfold configuration. The CV approach is attractive because it uses a simple ideal gas law to compute the pressure change in each $\mathrm{CV}$, and then uses the pressure to drive an incremental finite element analysis of the opening structure. Additional simulations were made with the Z-fold configurations to evaluate the effects of the rate of inflation, the presence of residual air in the packaged configuration, and the effect of deploying in the presence of gravity.

The deployment simulations with different inflation rates indicate that a low inflation rate may not yield a desirable deployment sequence. With the low inflation rate, the control volumes may require unacceptably high pressures to open the fold lines (kinks) and obtain a fully deployed structure. It was also determined that the pressure and amount of inflation gas required for full deployment are not simply related to the inflation rate. Deployment results for a fast inflation rate can not be scaled to predict the structural behaviors at a slow inflation rate.

A very small amount of residual air can dynamically open the control volumes, pushing them apart from each other. This helps prevent the "kink formation" and results in a much shorter deployment time with a lower deployment pressure.

Gravitational forces can impair the opening of the fold lines in a packaged tube. This is similar to the case of a deployment at a low inflation rate. High deployment pressures (over those required in space) may be required. Thus, deployment test results obtained on the ground will not fully predict a deployment in space. However, ground tests can be used to validate an inflation simulation model by correlating the computational results with the ground test data. The on ground validated model can then be used to better understand deployment in space.

\section{References}

1. Mclnnes, C. R., Solar Sailing Technology, Dynamics and Mission Applications, ISBN 185233-102-X, Praxis Publishing Ltd, Chichester, UK, 1999 .

2. Chmielewski, A. B., and Moore, C., "The Gossamer Initiative," IEEE paper 0-7803-5846-5, January 2000 .

3. Steele, C. R., and Fay, J. P., "Inflation of Rolled Tube," IUTAM-IASS Symposium on Deployable Structures: Theory and Applications: Proceedings of the IUTAM Symposium held in Cambridge, U.K., 6-9 September 1998 /edited by S. Pellegrino and S.D. Guest, pp. 393-403.

4. Haug, E., Protard, J. B., Milcent G., Herren, A., and Brunner, O., "The Numerical Simulation of the Inflation Process of Space Rigidized Antenna Structures," Proc. Internat. Conf.: Spacecraft Structures and Mechanical Testing, Noordwijk, The Netherlands, 24-26, April 1991, ESA SP-321, October 1991, pp. 861-869.

5. Salama, M., Kuo, C. P., Lou, M., "Simulation of Deployment Dynamics of Inflatable Structures," AIAA Journal, Vol. 38, No. 12, 2000, pp. 2277 2283.

6. Hoffmann, R., Pickett, A. K., Ulrich, D., Haug, E., Lasry, D., and Clinckemaillie, J., " A Finite Element Approach to Occupant Simulation - The PAM-CRASH Airbag Model," SAE Paper 890754, SAE Transactions: Journal of Passenger Cars, Vol. 98, 1989, pp. 885-893

7. LS-DYNA, Theoretical Manual, Complied by Hallquist, J. O., Livermore Software Technology Corporation, May 1998.

8. Wang, J. T., and Nefske, O. J., “A NEW CAL3D Airbag Inflation Model," SAE paper 880654, 1988.

9. Belytschko, T., Liu, W. K., and Moran B., Nonlinear Finite Elements for Continua and structures, John Wiley \& Sons, Inc., West Sussex, England, ISBN 0-471-98773-5, 2000.

10. Freeland, R. E., Bilyeu, G. D., Veal, G. R., Steiner, M. D., and Carson, D. E., "Large Inflatable Deployable Antenna Flight Experiment Results," 
ACTA Astronautica, Vol. 41, Nos 4-10, 1997, pp. 267-277.

11. Belytschko, T., Lin, J. and Tsay, C. S., "Explicit Algorithms for the Nonlinear Dynamics of Shells,"
Computer Methods in Applied Mechanics and Engineering, Vol. 42, 1984, pp. 225-251.

Table 1. Inflation time, internal pressure, and total mass of the inflation gas for Z-fold deployment simulations.

\begin{tabular}{|c|c|c|c|c|c|c|}
\hline \multirow{2}{*}{\multicolumn{2}{|c|}{$\begin{array}{c}\text { Inflation rate, } \\
\qquad d m / d t \\
(1 \mathrm{bm} / \mathrm{sec})\end{array}$}} & \multirow{3}{*}{$\begin{array}{c}\text { Inflation time } * * *, \\
\tau \\
(\mathrm{sec}) \\
\\
1.38\end{array}$} & \multicolumn{3}{|c|}{$\begin{array}{c}\text { Pressure } \\
(\mathrm{psi})\end{array}$} & \multirow{3}{*}{$\begin{array}{l}\text { Total Inflation } \\
\text { Mass**, } \\
m(\tau) \\
(\mathrm{lbm}) \times 10^{-3} \\
9.52\end{array}$} \\
\hline & & & \multirow{2}{*}{$\begin{array}{c}\begin{array}{c}\text { First } \\
\mathrm{CV}\end{array} \\
9.26\end{array}$} & \multirow{2}{*}{$\begin{array}{l}\begin{array}{c}\text { Last } \\
\mathrm{CV}\end{array} \\
9.23\end{array}$} & \multirow{2}{*}{$\begin{array}{c}\begin{array}{c}\text { Ave. of } \\
\text { all CVs }\end{array} \\
9.24\end{array}$} & \\
\hline $0.01 \times t$ & $4-\mathrm{CVs}$ & & & & & \\
\hline & $5-\mathrm{CVs}$ & 0.98 & 5.31 & 5.27 & 5.28 & 4.80 \\
\hline & 8-CVs & 0.75 & 3.29 & 3.25 & 3.26 & 2.81 \\
\hline \multirow[t]{3}{*}{$0.1 \times t$} & 4-CVs & 0.19 & 2.30 & 2.13 & 2.24 & 1.81 \\
\hline & $5-\mathrm{CVs}$ & 0.22 & 3.11 & 2.75 & 2.86 & 2.42 \\
\hline & 8-CVs & 0.20 & 2.57 & 2.20 & 2.33 & 2.00 \\
\hline \multirow[t]{3}{*}{$1.0 \times t$} & 4-CVs & 0.085 & 5.27 & 3.69 & 4.50 & 3.61 \\
\hline & 5-CVs & 0.085 & 6.47 & 3.53 & 4.41 & 3.61 \\
\hline & 8-CVs & 0.080 & 6.09 & 2.62 & 3.75 & 3.20 \\
\hline
\end{tabular}

* Time step, $\Delta t=8.35 \times 10^{-6}$ seconds

** $\tau=$ time at full deployment 


\section{$\underline{\text { Square solar sail }}$}

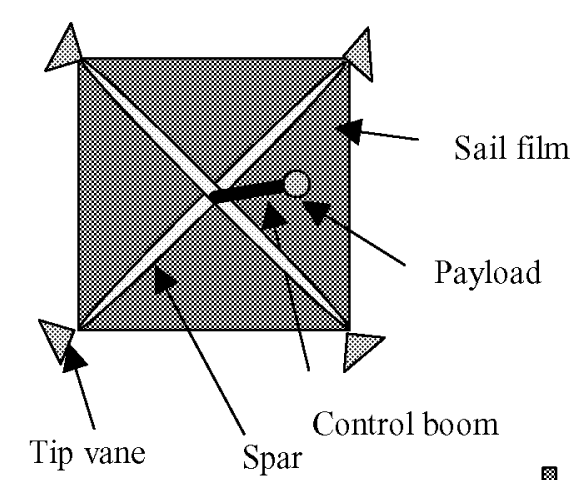

\section{Disc solar sail}

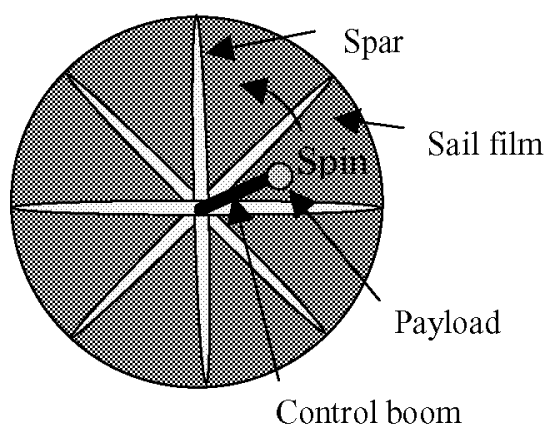

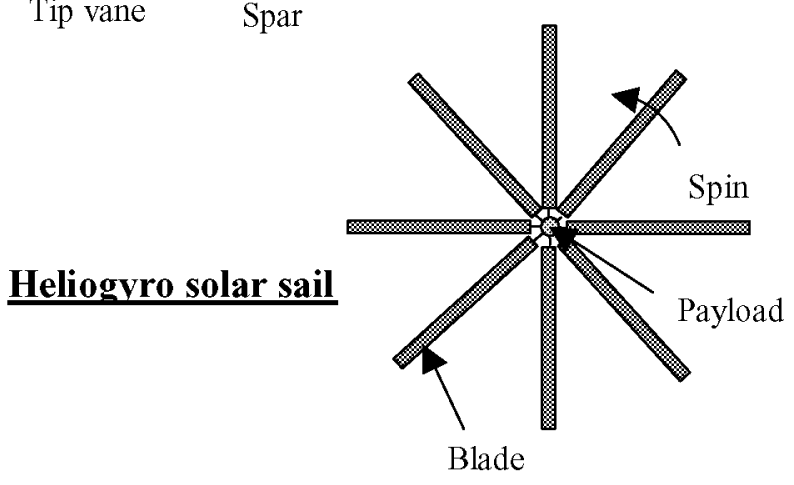

Figure 1. Three solar sail configurations (Ref. 1).

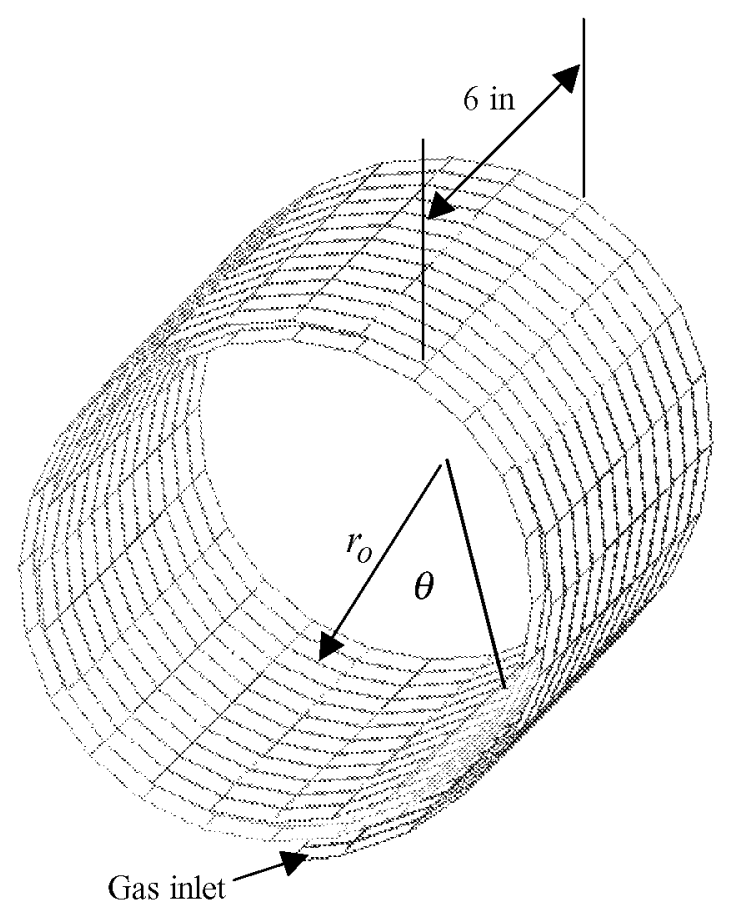

Figure 2. Finite element model of a coiled tube. 


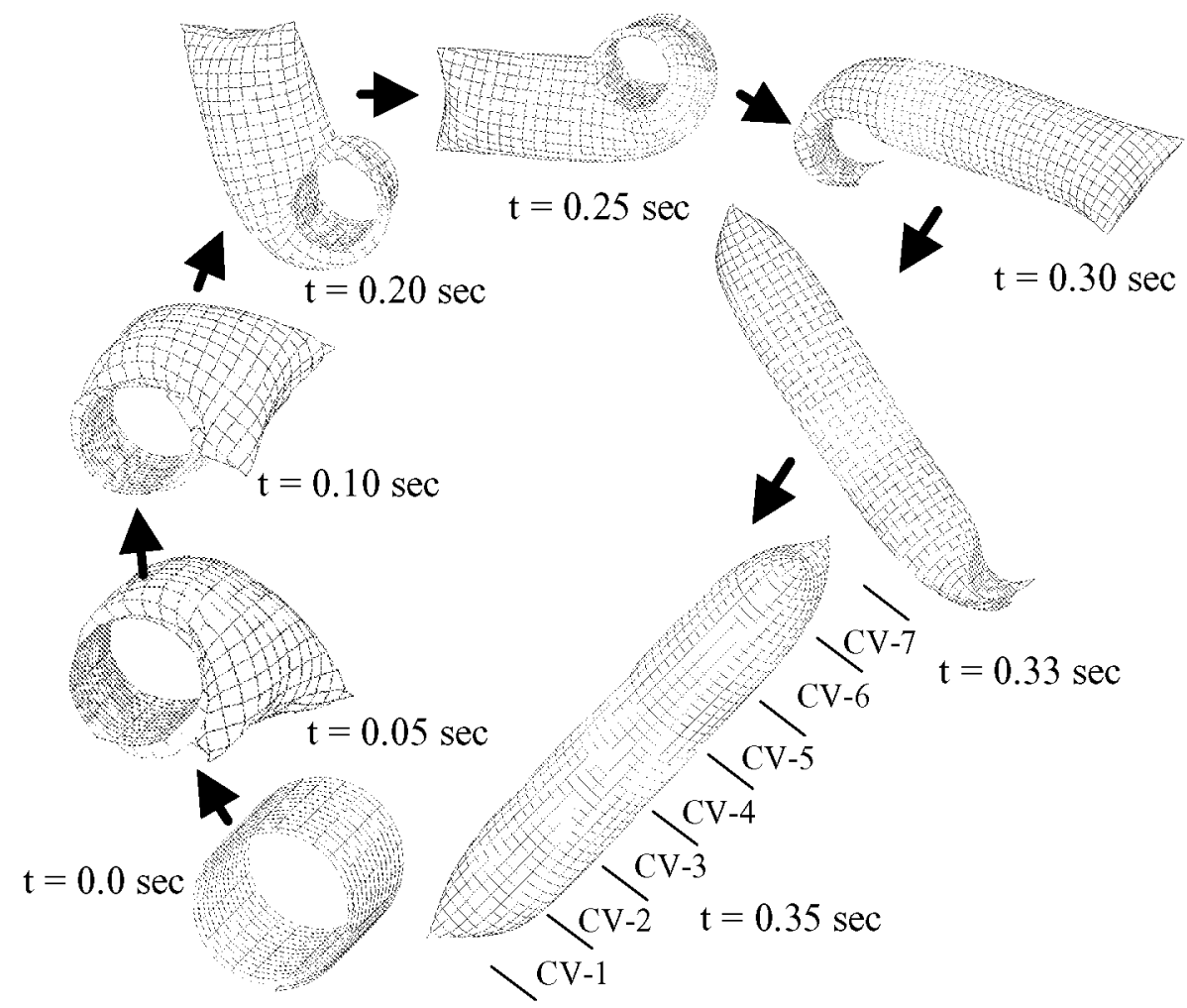

Figure 3. Coiled tube at different deployment stage.

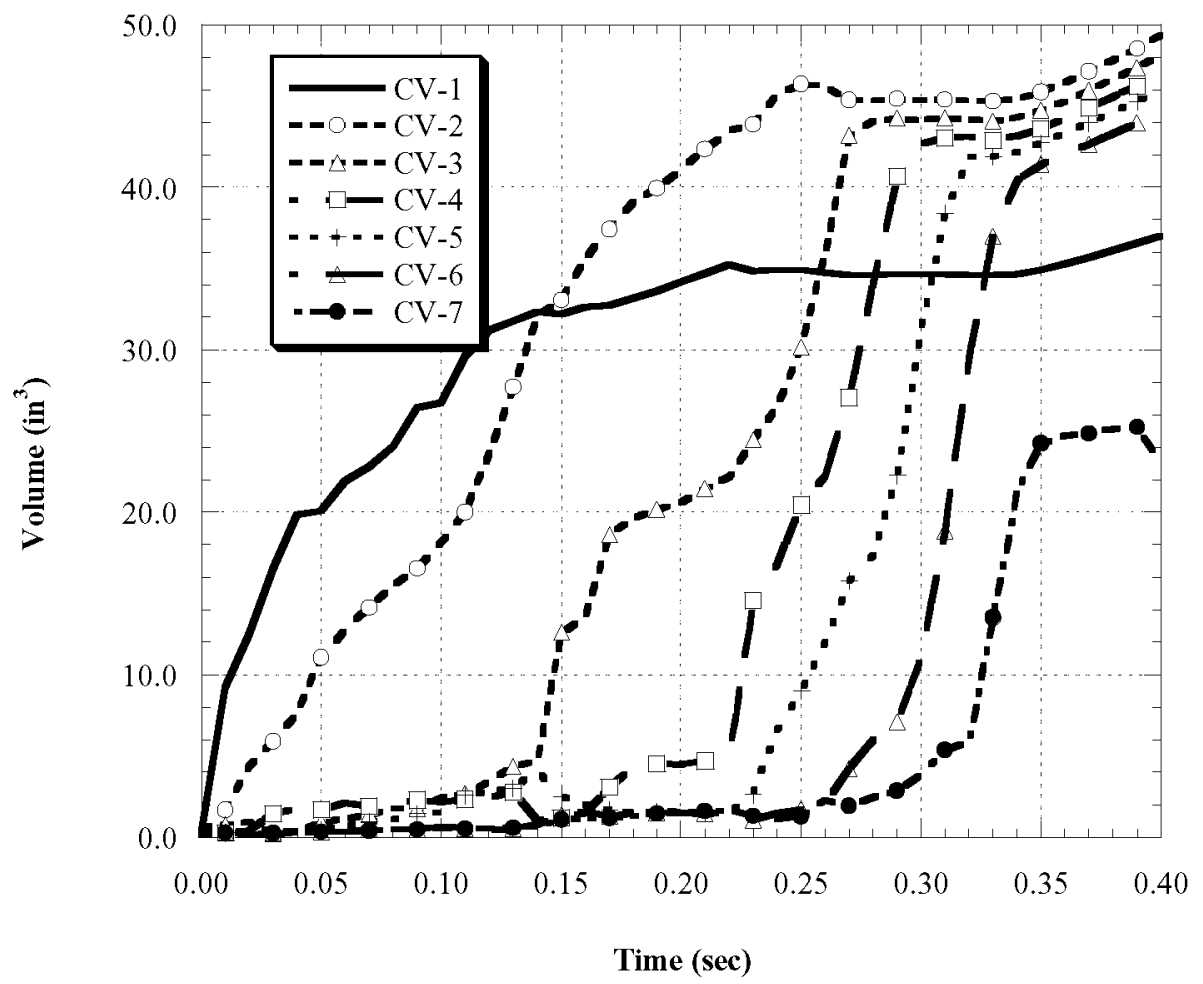

Figure 4. Volume of each control volume (CV) as a function of time. 


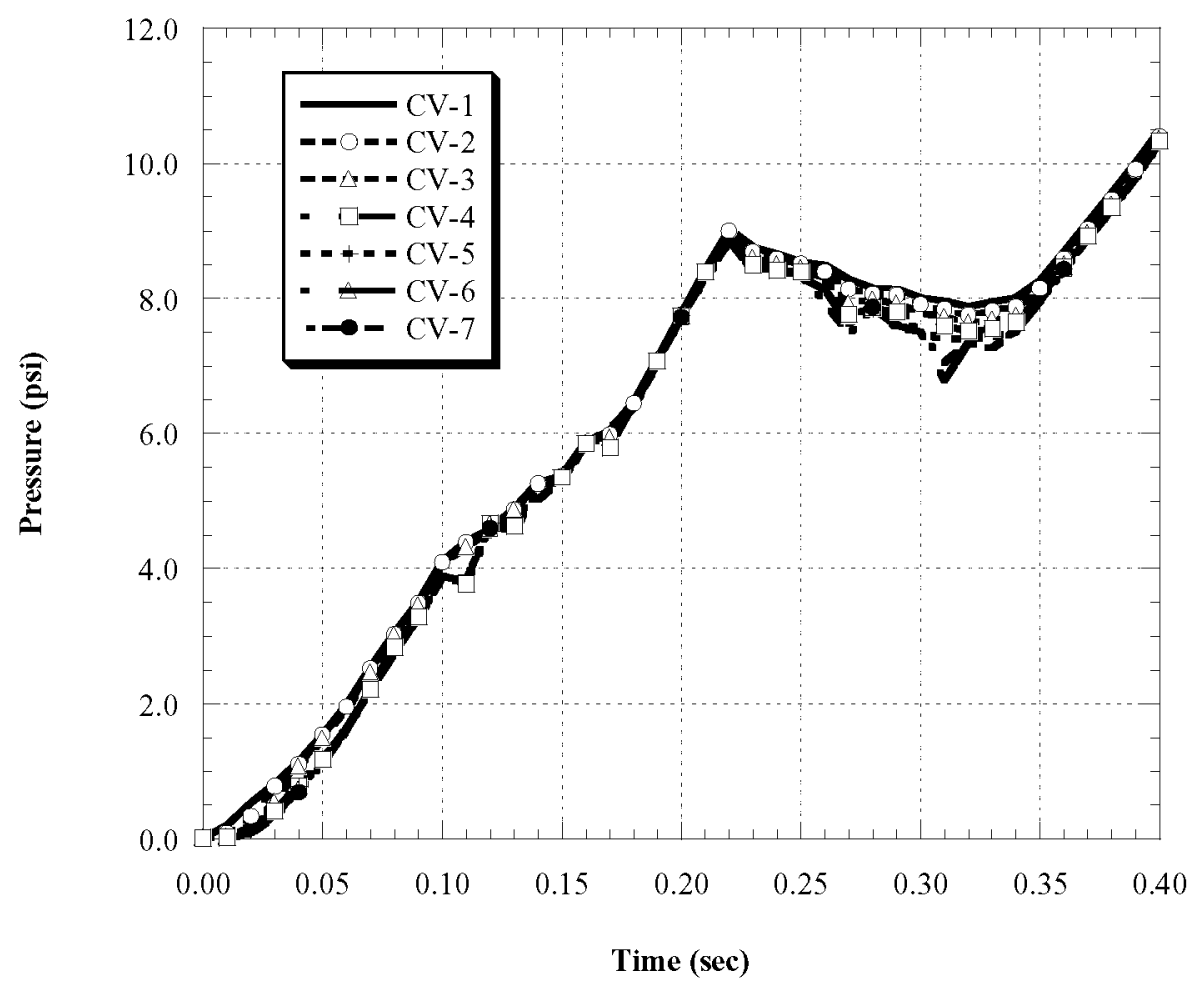

Figure 5. Pressure of each control volume (CV) as a function of time.

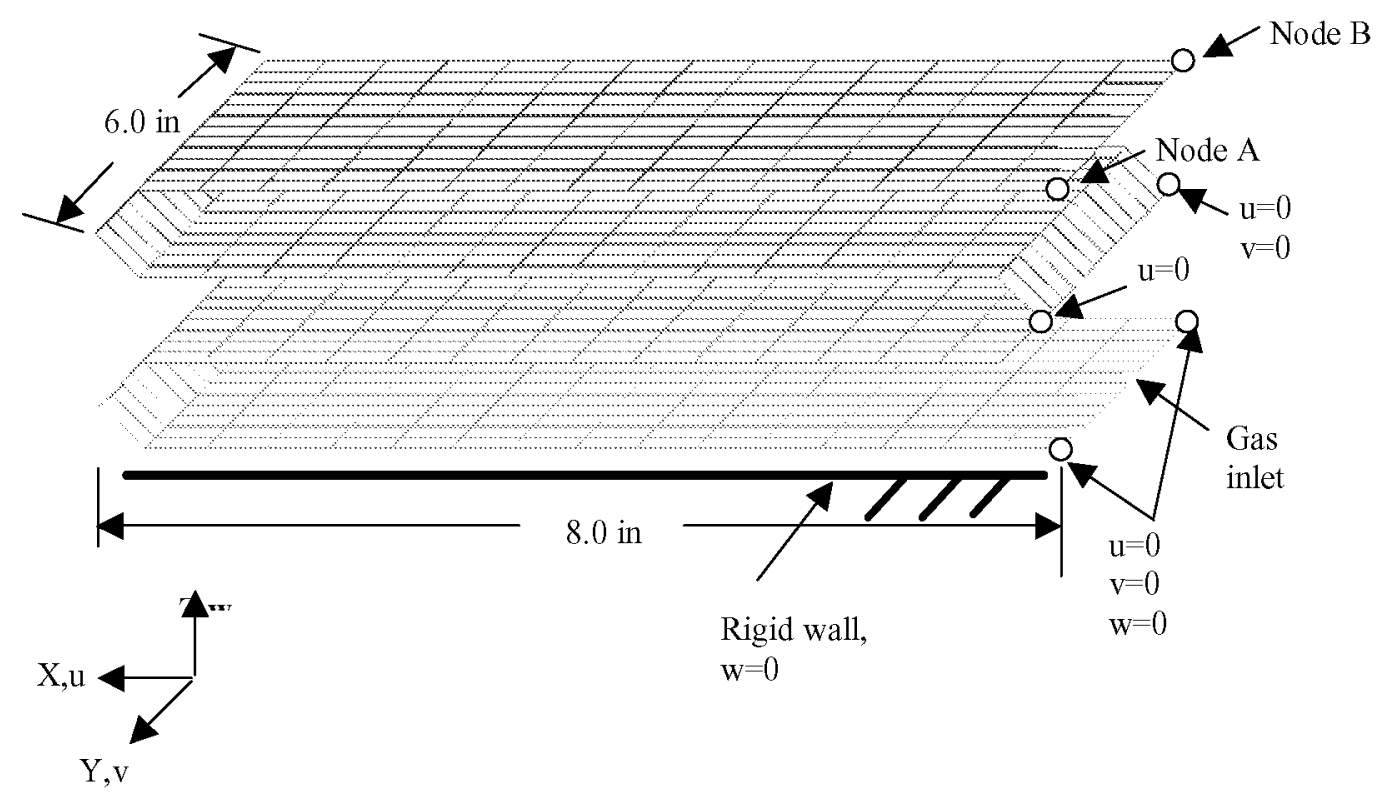

Figure 6. Finite element model of a Z-folded tube. 

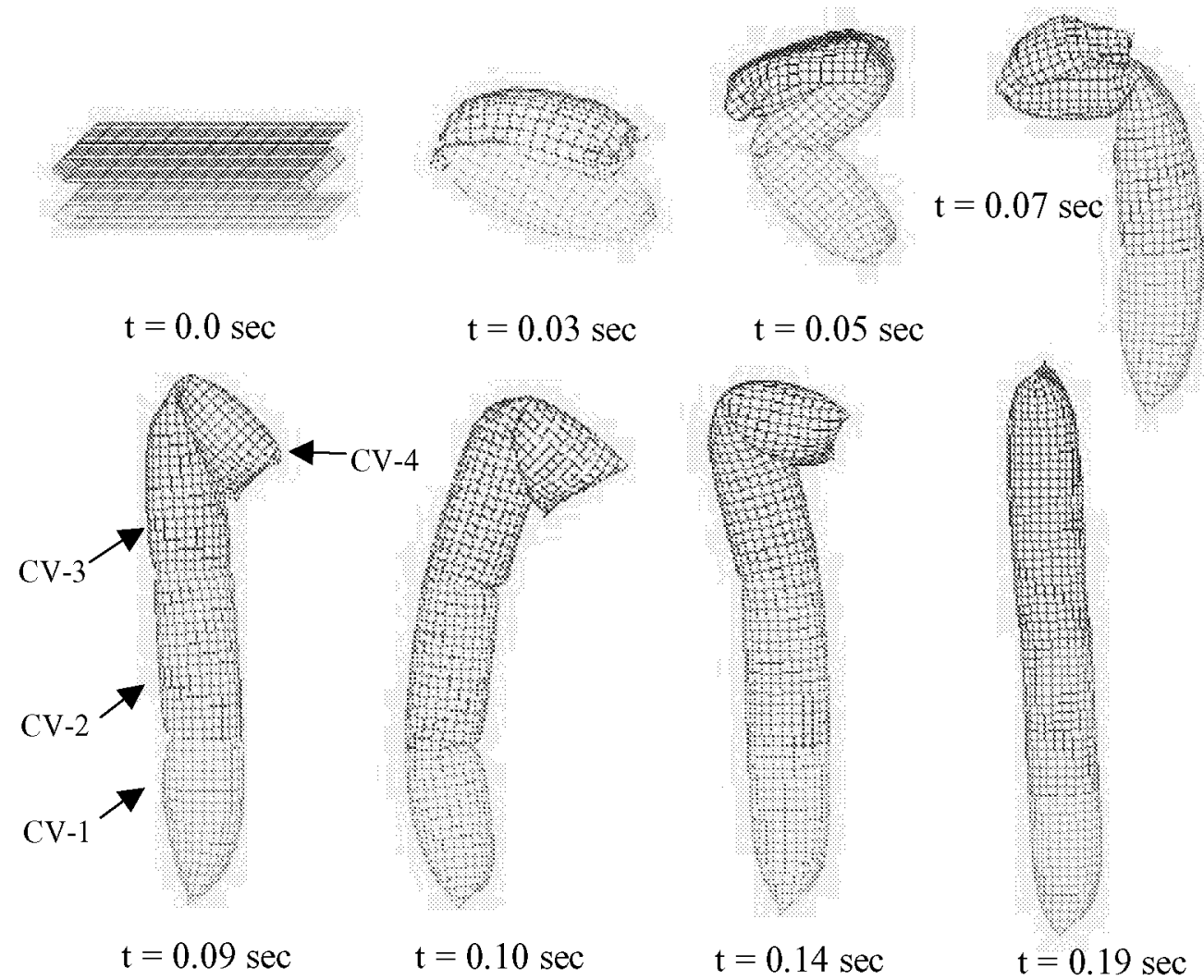

Figure 7. Z-folded tube at different deployment stages.

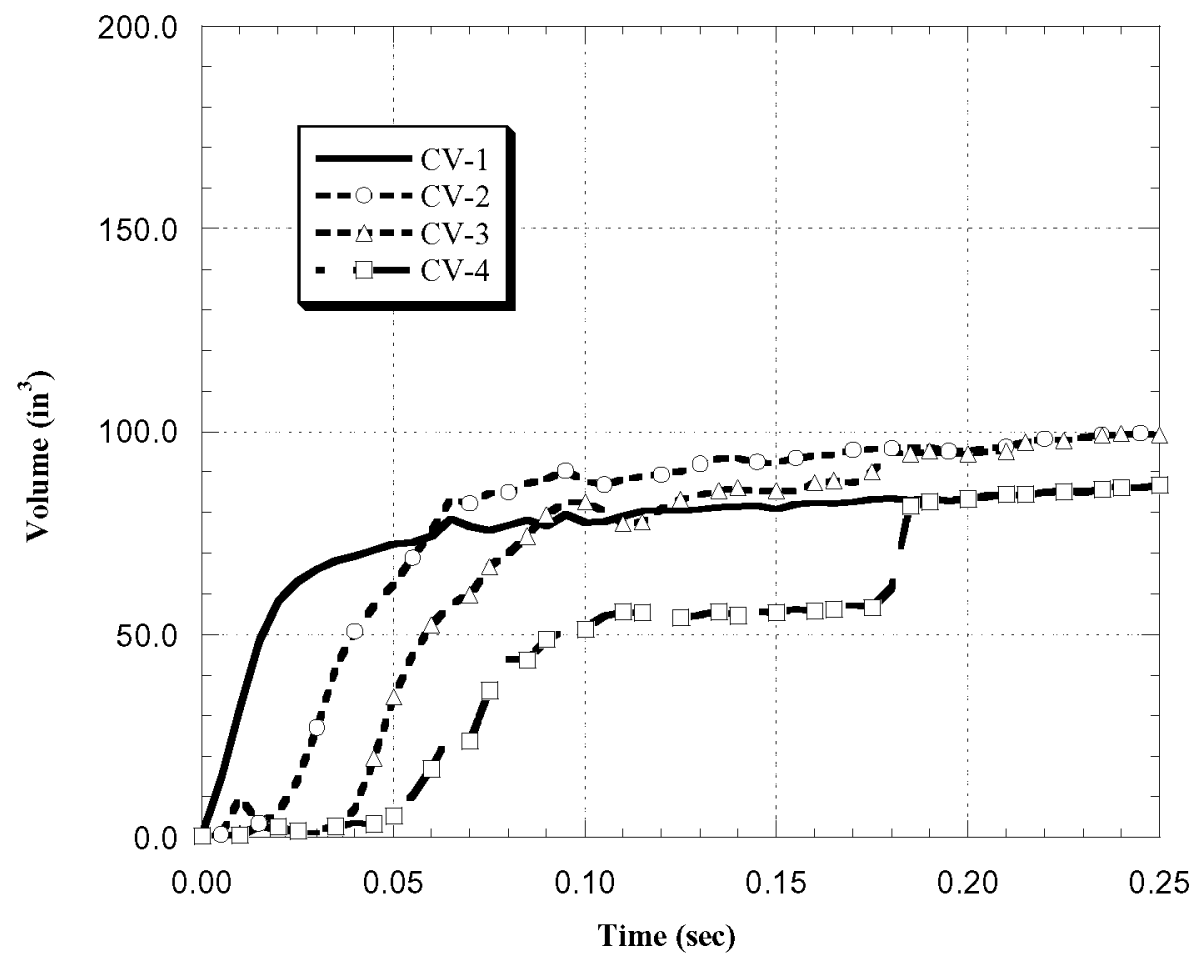

Figure 8. Volume of each control volume (CV) as a function of time. 


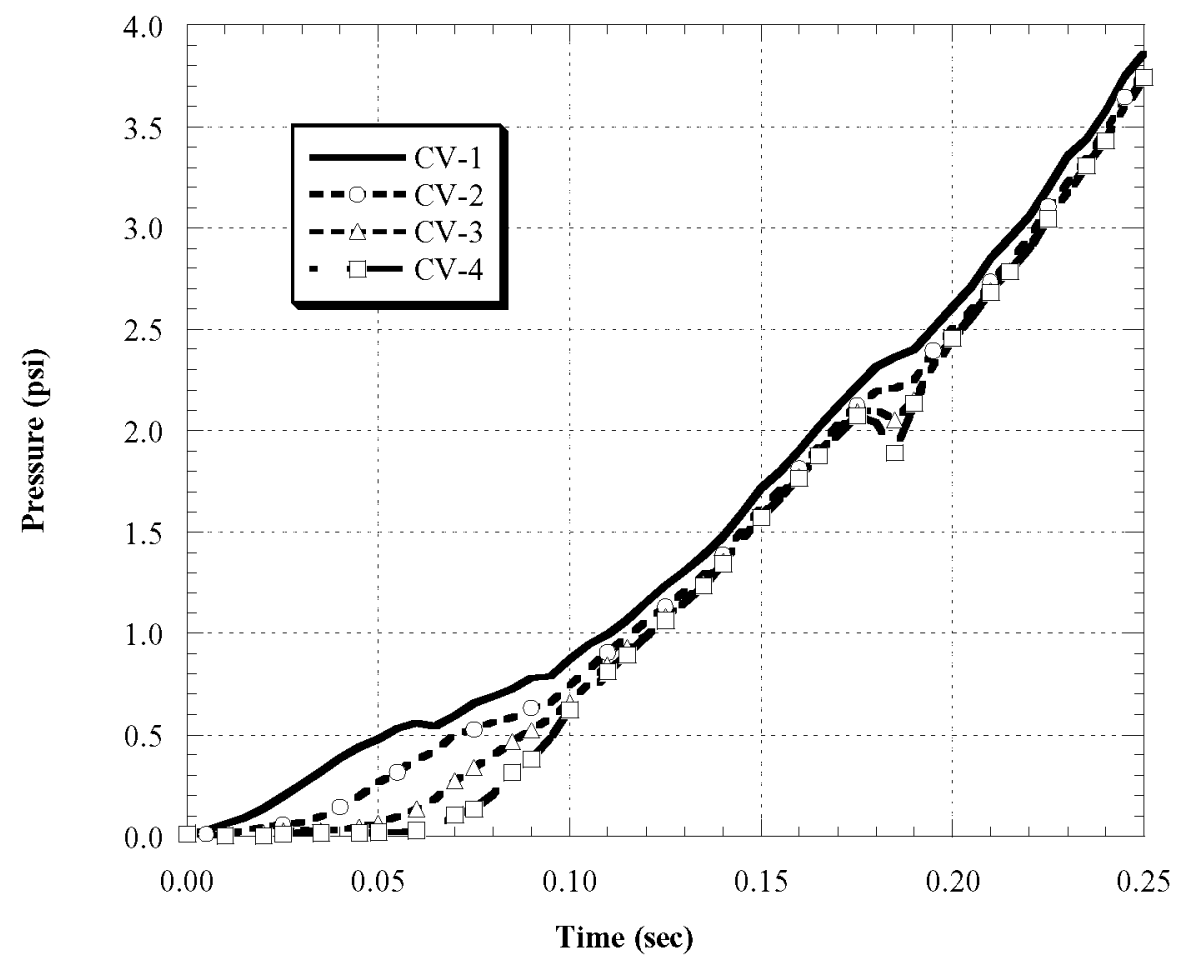

Figure 9. Pressure of each control volume (CV) as a function of time.
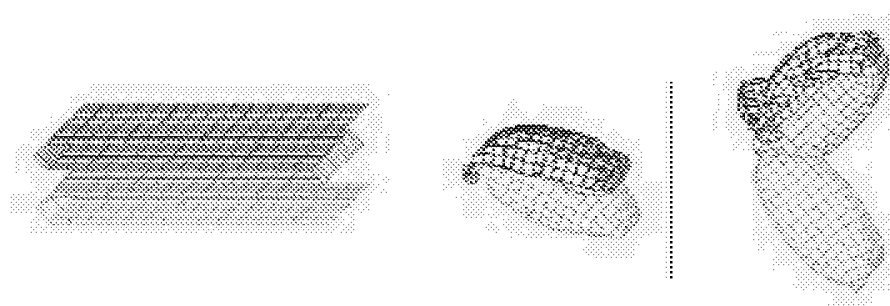

$\mathrm{t}=0.0 \mathrm{sec}$
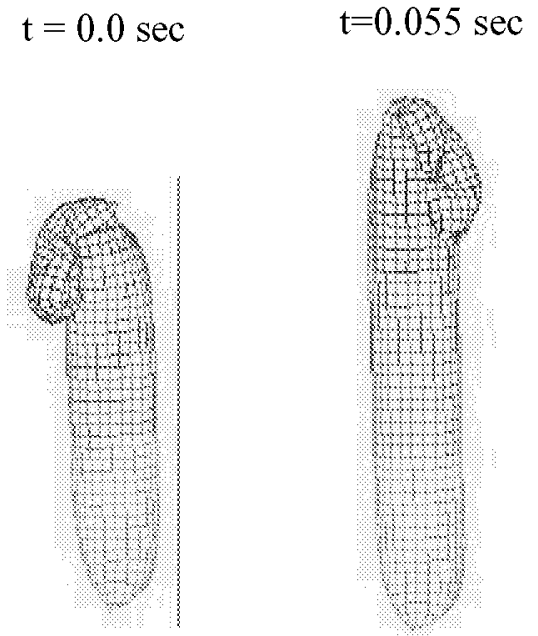

$\mathrm{t}=0.30 \mathrm{sec}$

$$
\mathrm{t}=0.40 \mathrm{sec}
$$
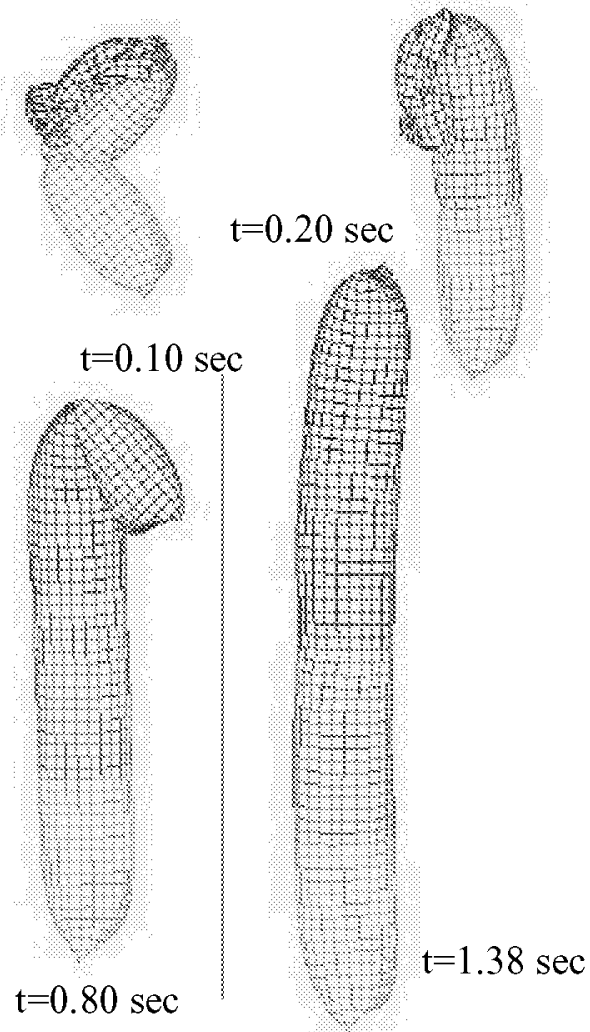

Figure 10. Different deployment stages of the Z-folded tube under a slow inflation rate. 


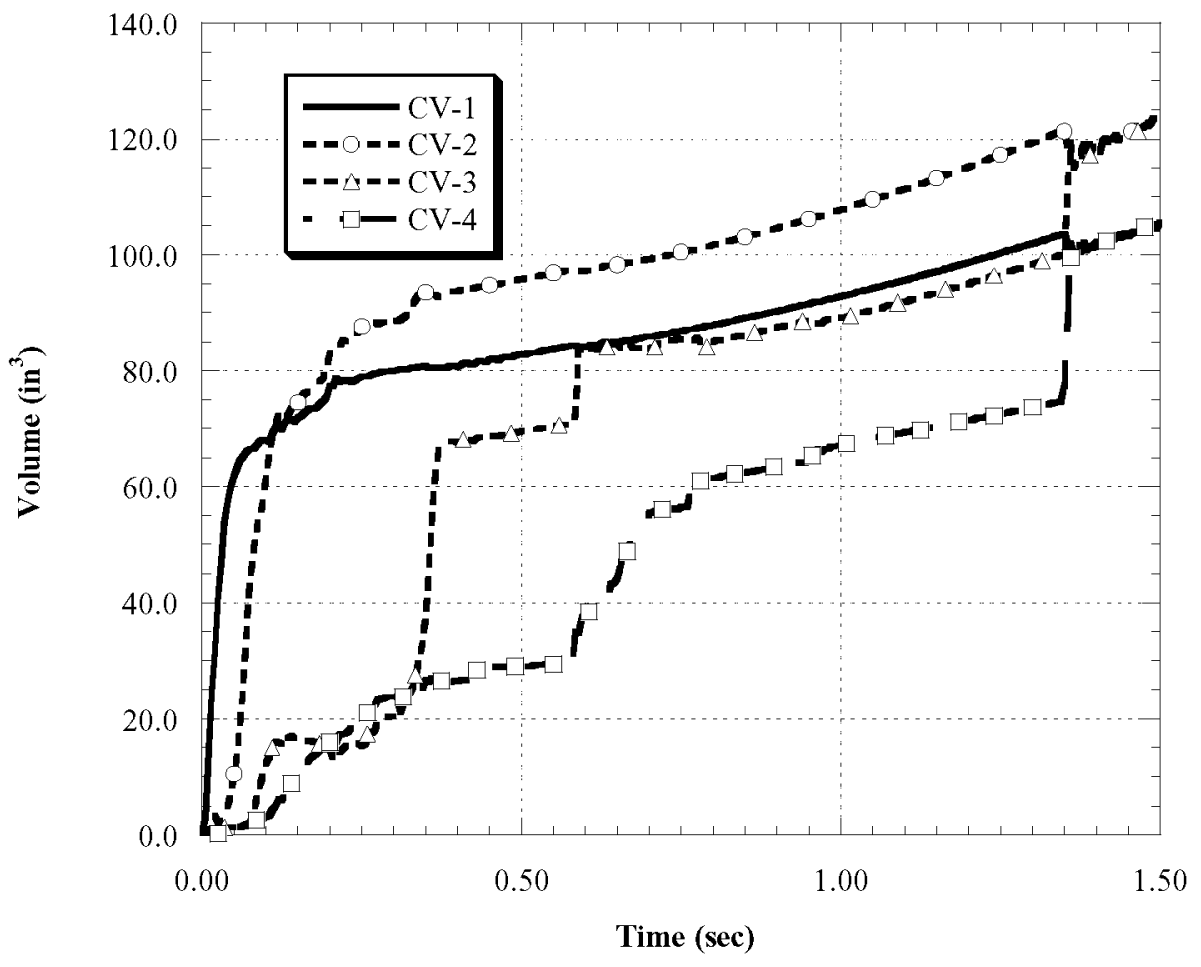

Figure 11. Volume of each control volume (CV) as a function of time for the slow inflation rate case.

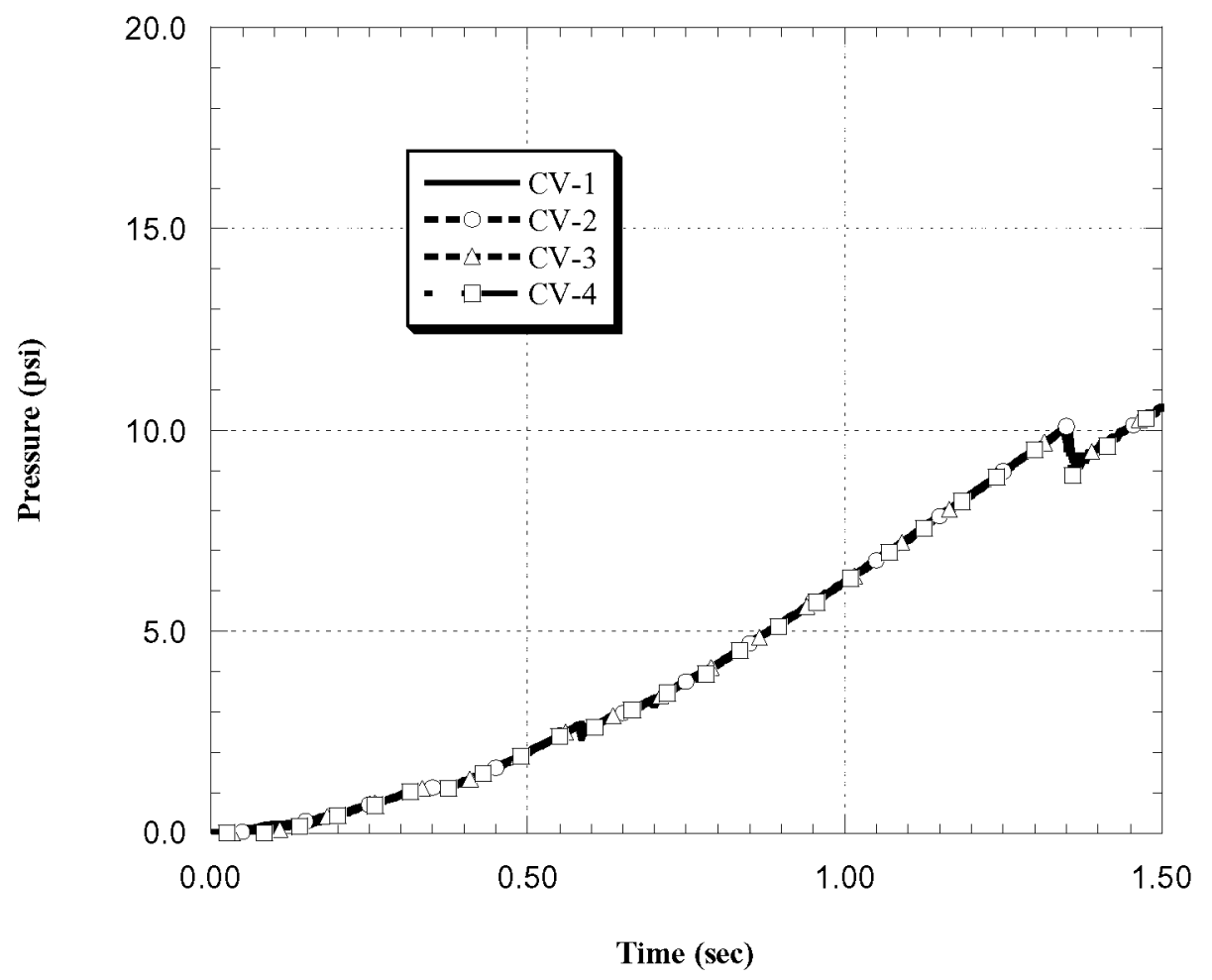

Figure 12. Pressure of each control volume (CV) as a function of time for the slow inflation rate case. 

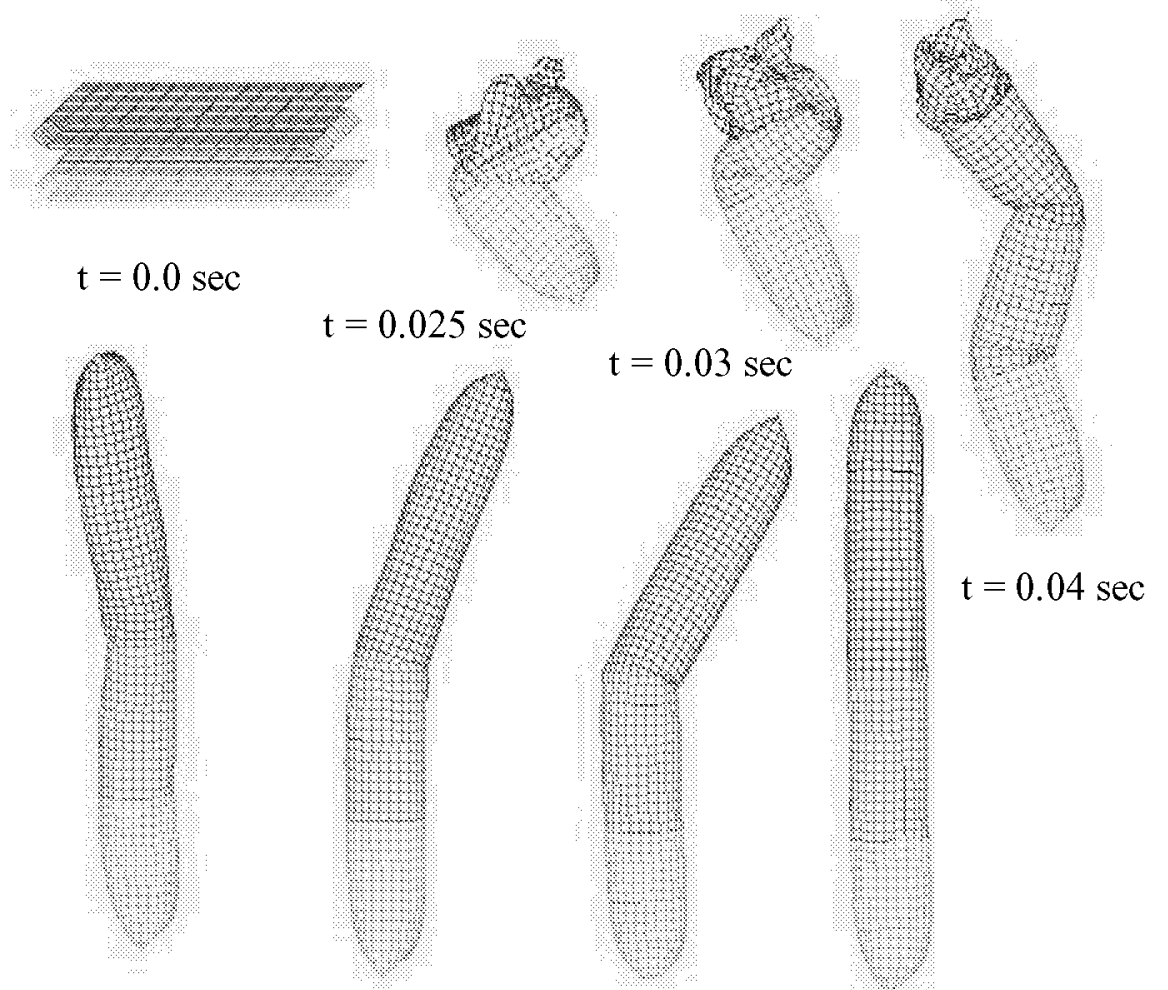

$\mathrm{t}=0.05 \mathrm{sec}$

$$
\mathrm{t}=0.06 \mathrm{sec} \quad \mathrm{t}=0.07 \mathrm{sec} \quad \mathrm{t}=0.085 \mathrm{sec}
$$

Figure 13. Different deployment stages of the Z-folded tube under a fast inflation rate.

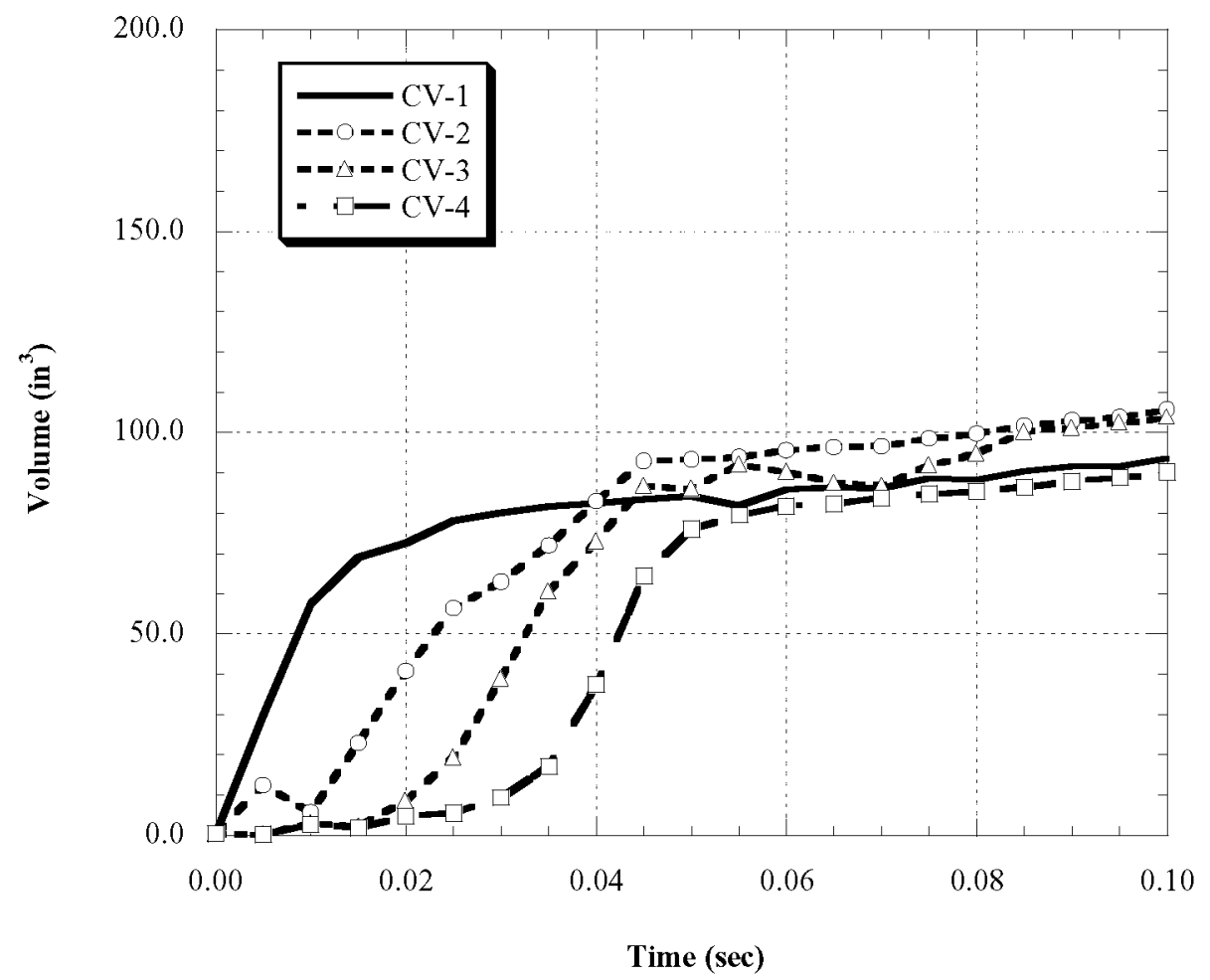

Figure 14. Volume of each control volume (CV) as a function of time for the fast inflation case. 


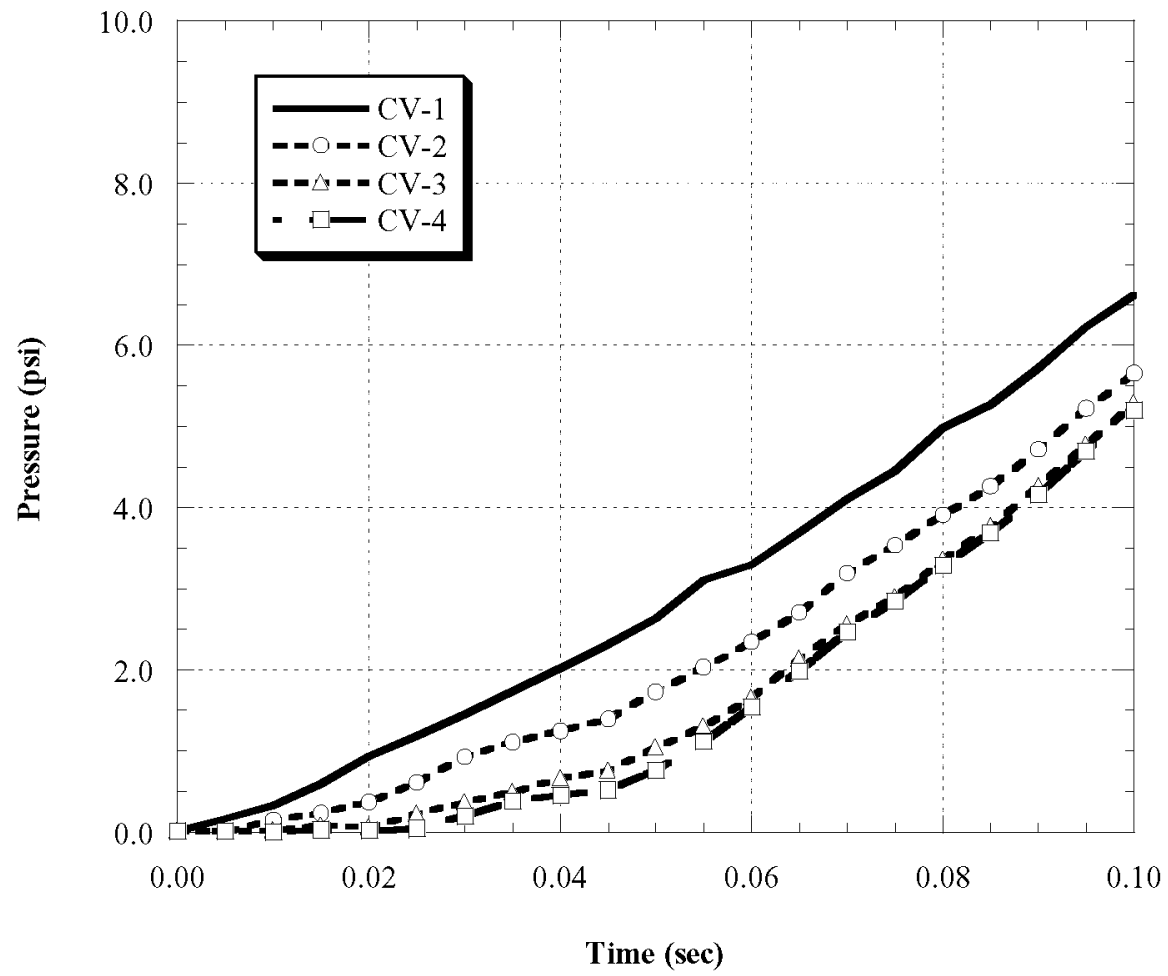

Figure 15. Pressure of each control volume (CV) as a function of time for the fast inflation case.
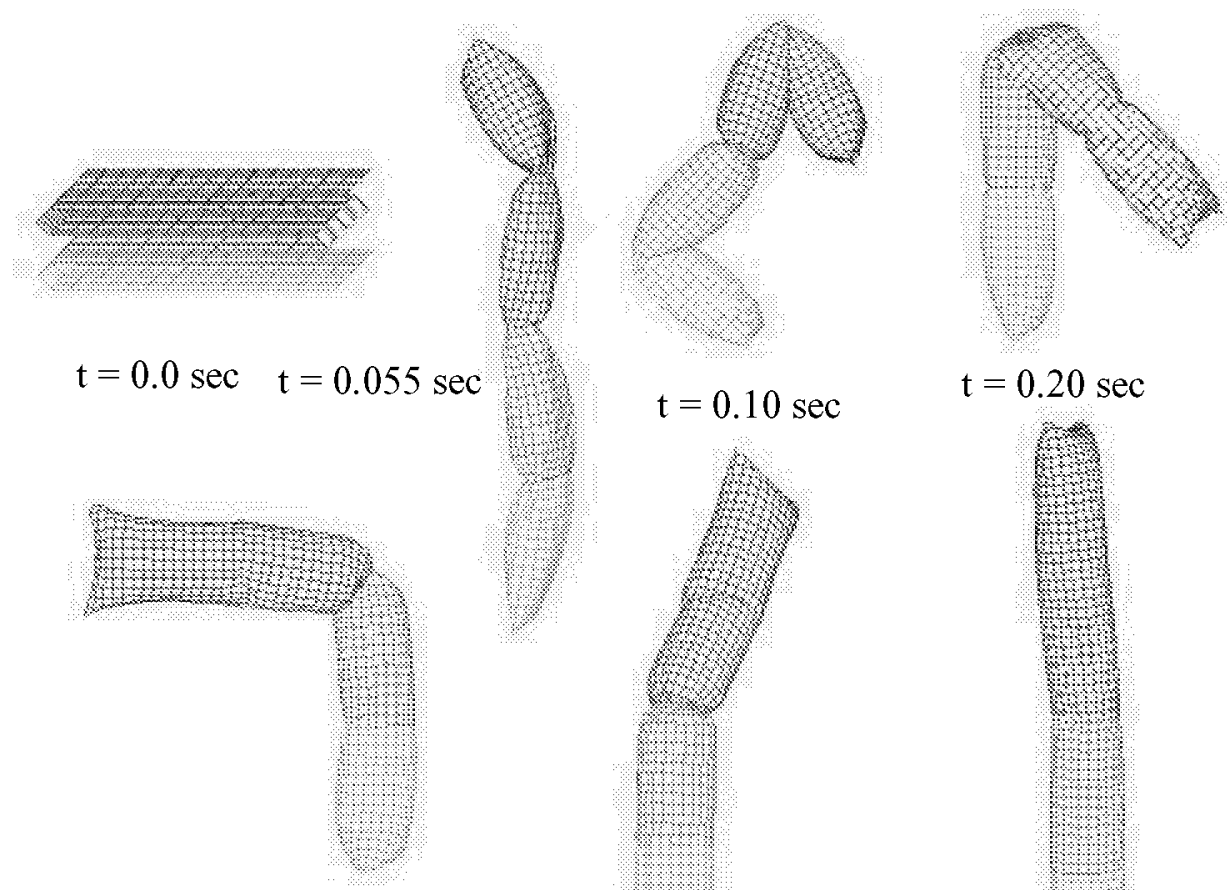

$\mathrm{t}=0.30 \mathrm{sec}$
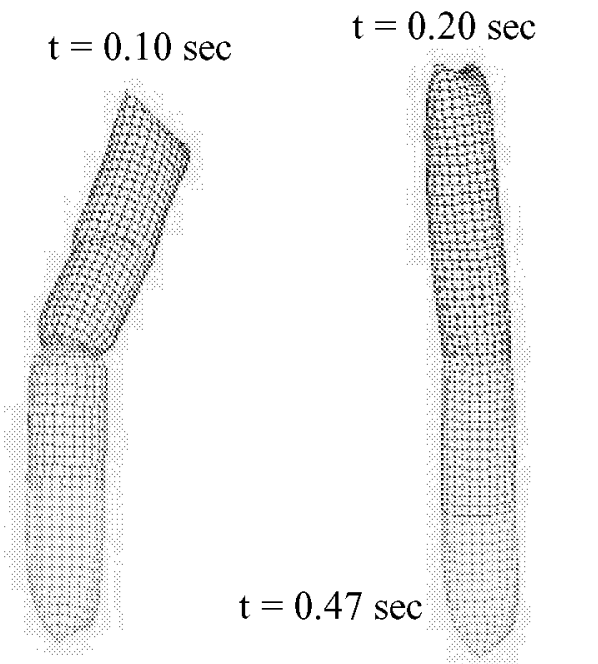

Figure 16. Different deployment stages of the Z-folded tube with residual air. 


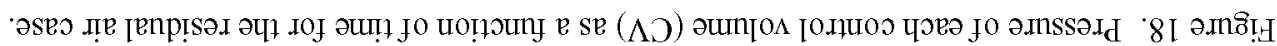

(əos) әщ!! L

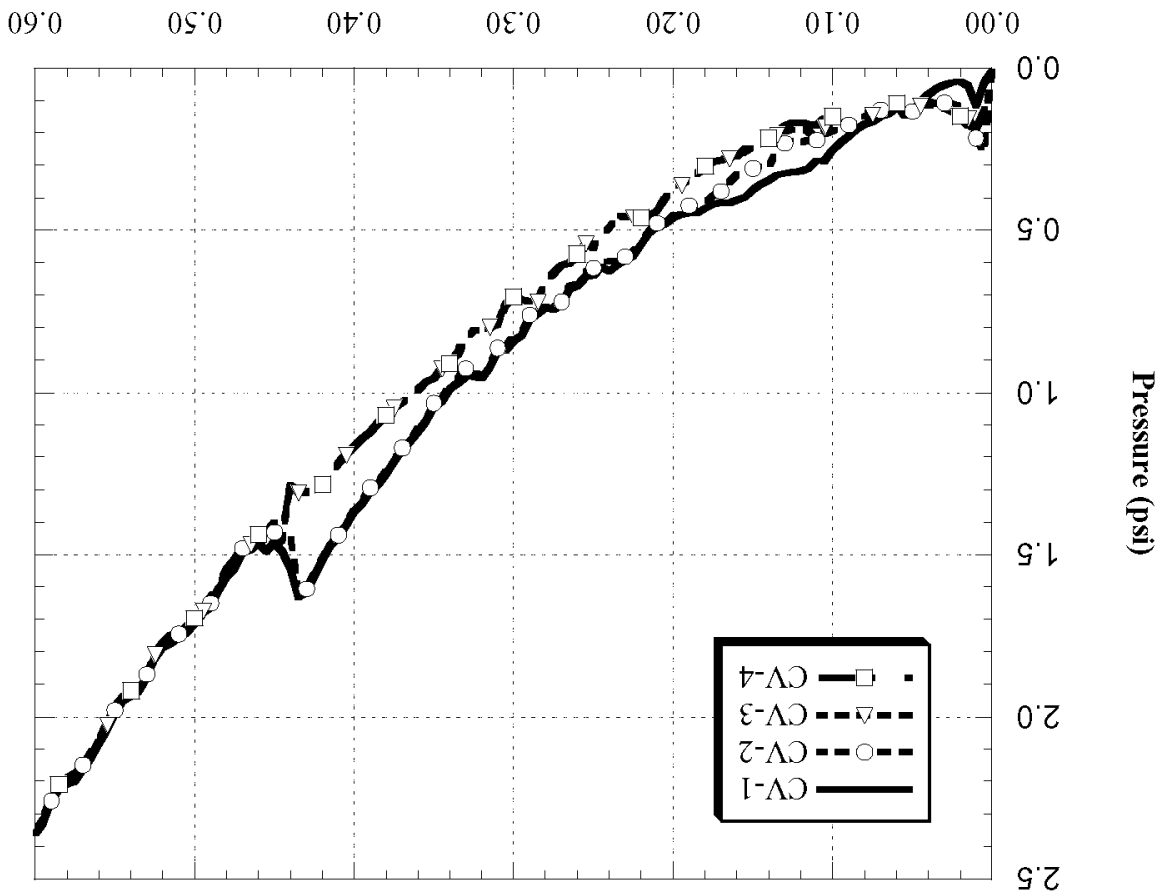

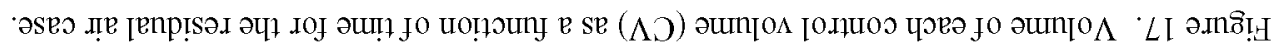

(aəs) วய!L

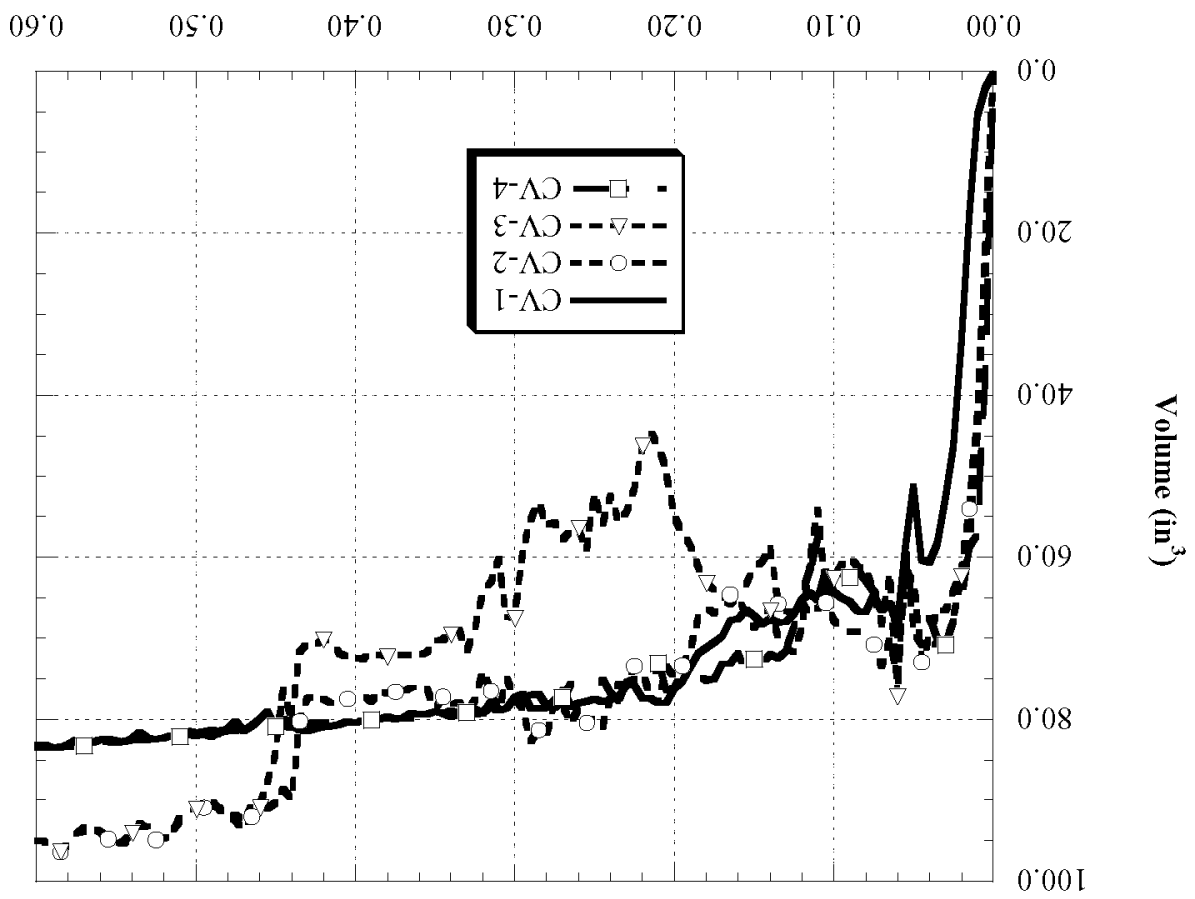




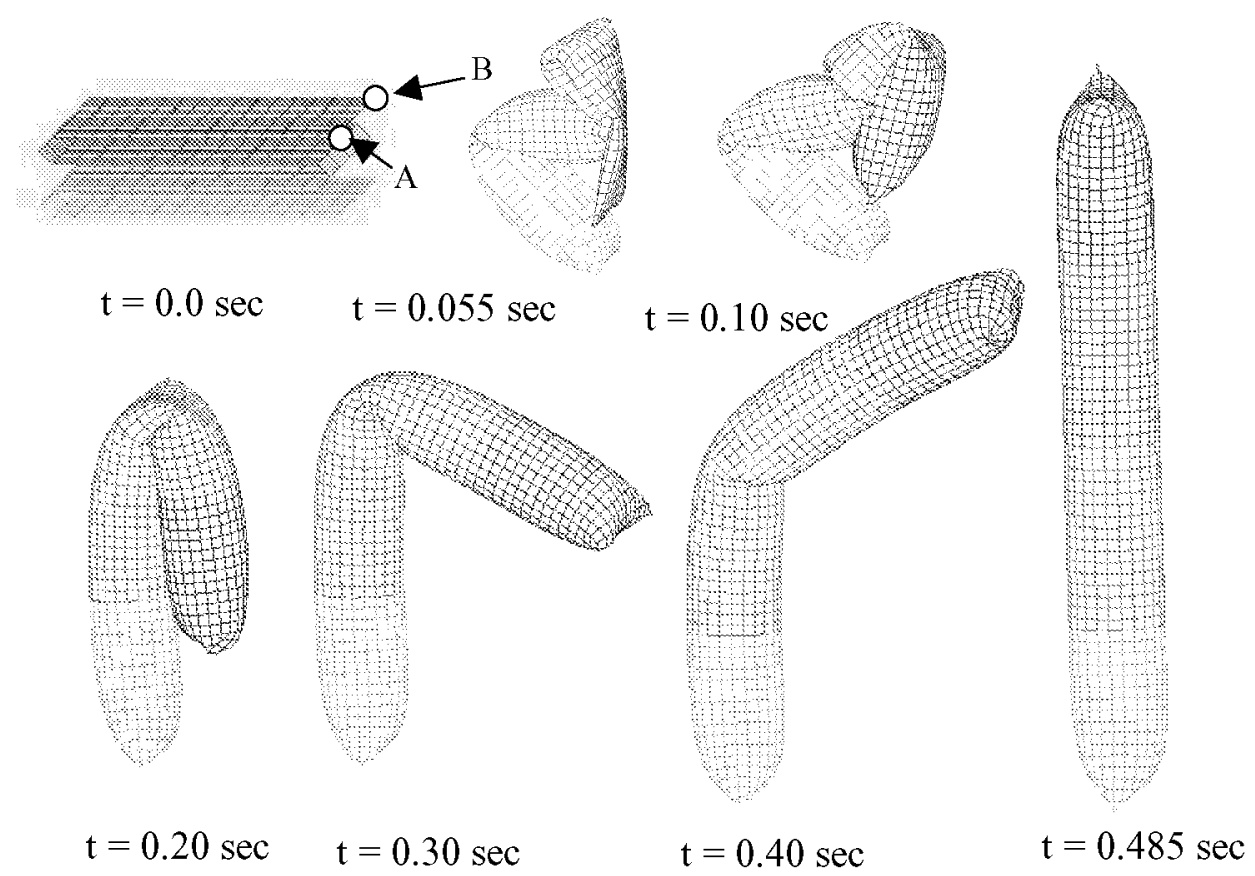

Figure 19. Different deployment stages of the Z-folded tube with end mass and no gravity.

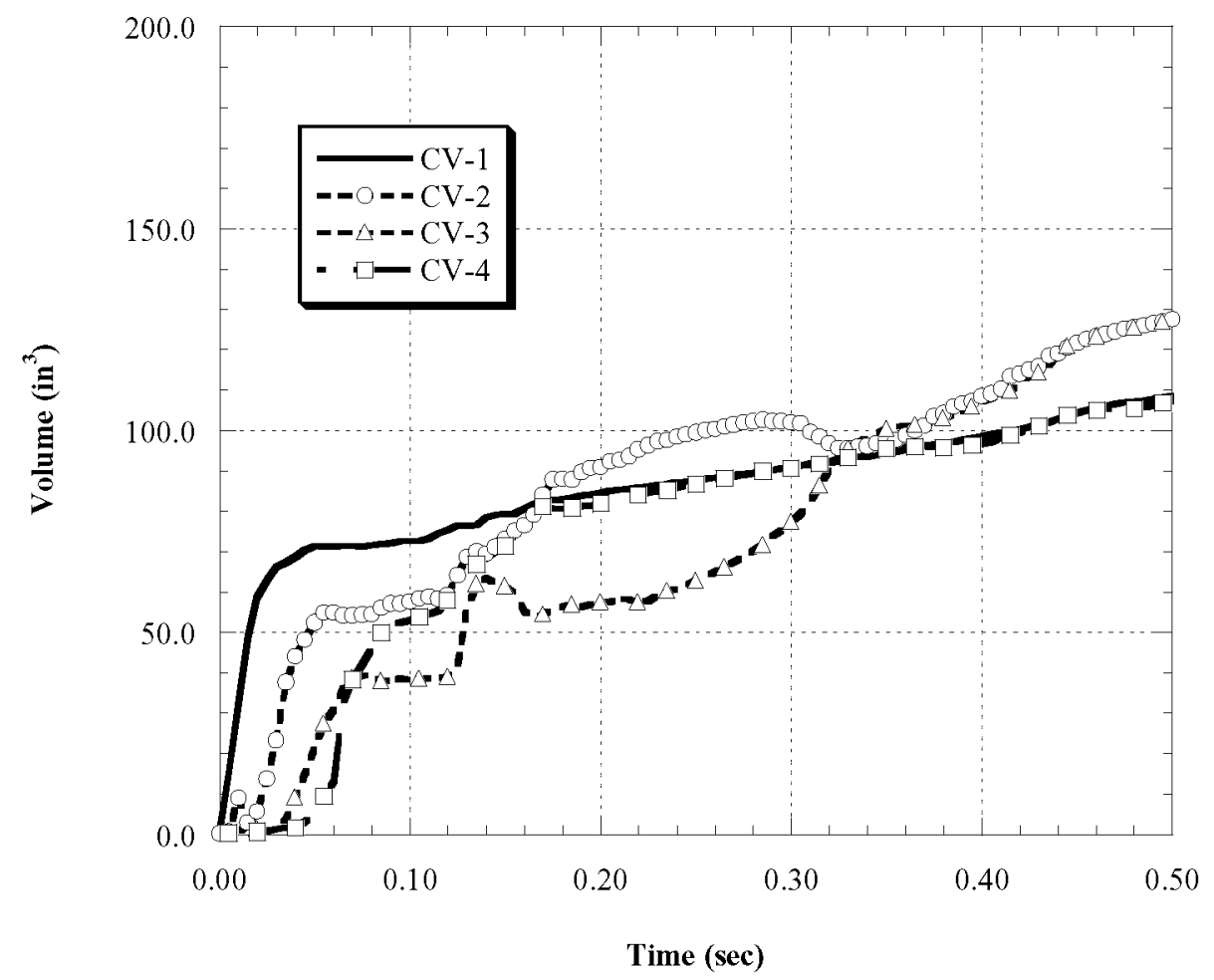

Figure 20. Volume of each control volume (CV) as a function of time for the no gravity case. 


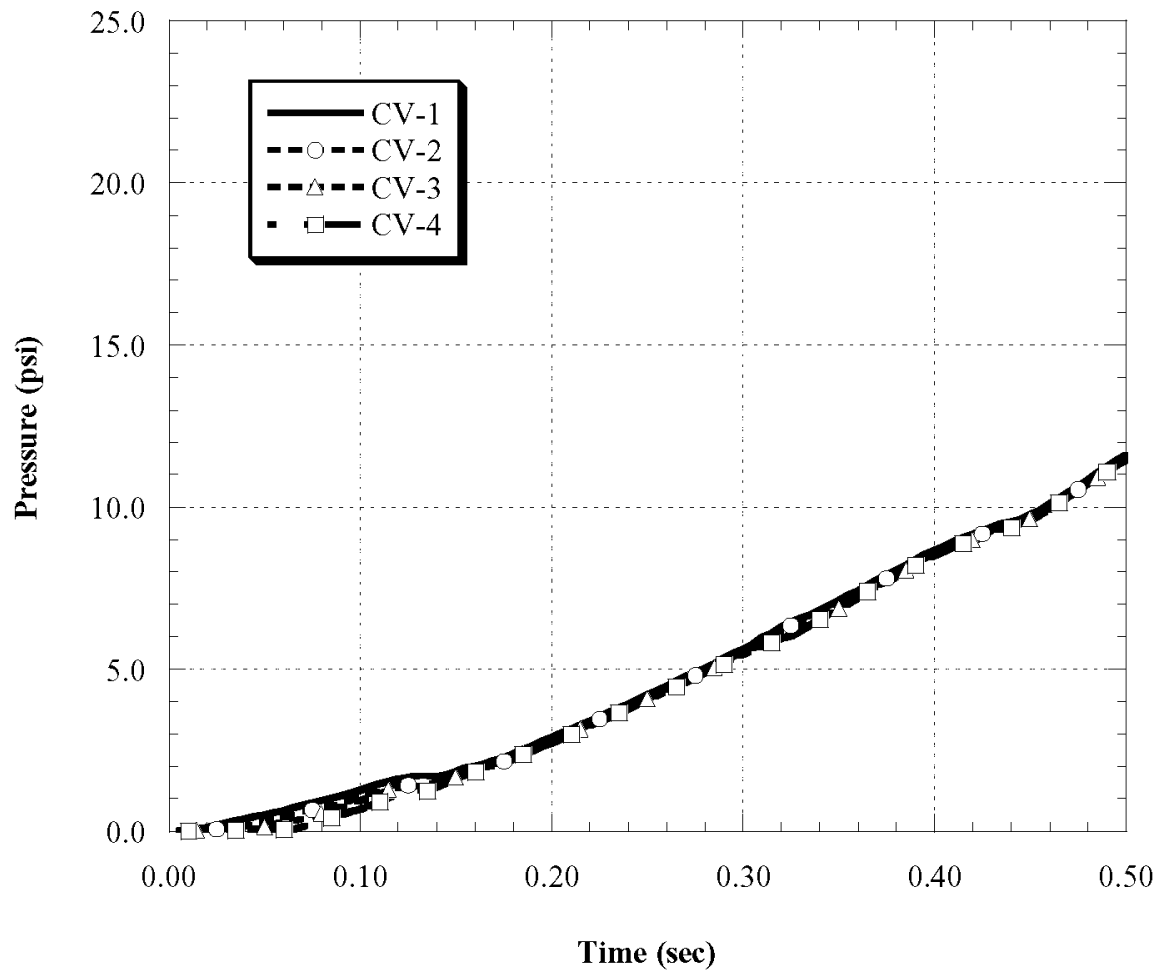

Figure 21. Pressure of each control volume (CV) as a function of time for the no gravity case.
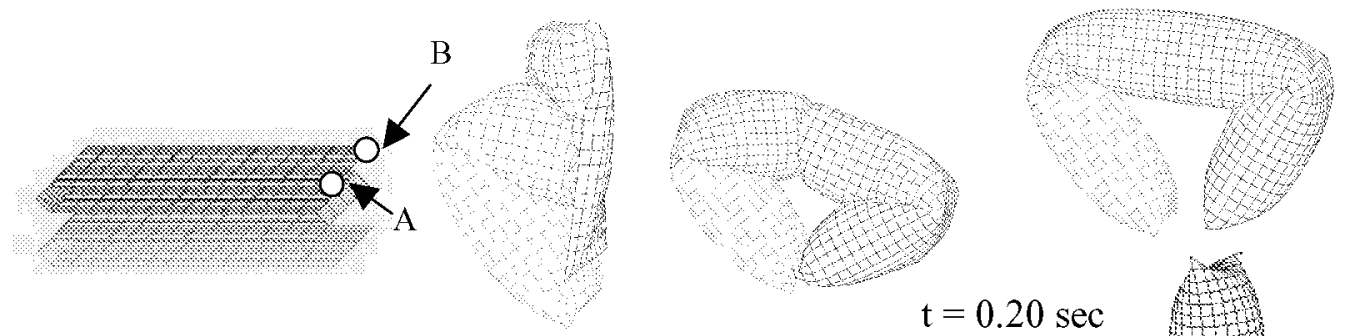

$$
\mathrm{t}=0.20 \mathrm{sec}
$$

$$
\mathrm{t}=0.0 \mathrm{sec} \quad \mathrm{t}=0.055 \mathrm{sec} \quad \mathrm{t}=0.10 \mathrm{sec}
$$
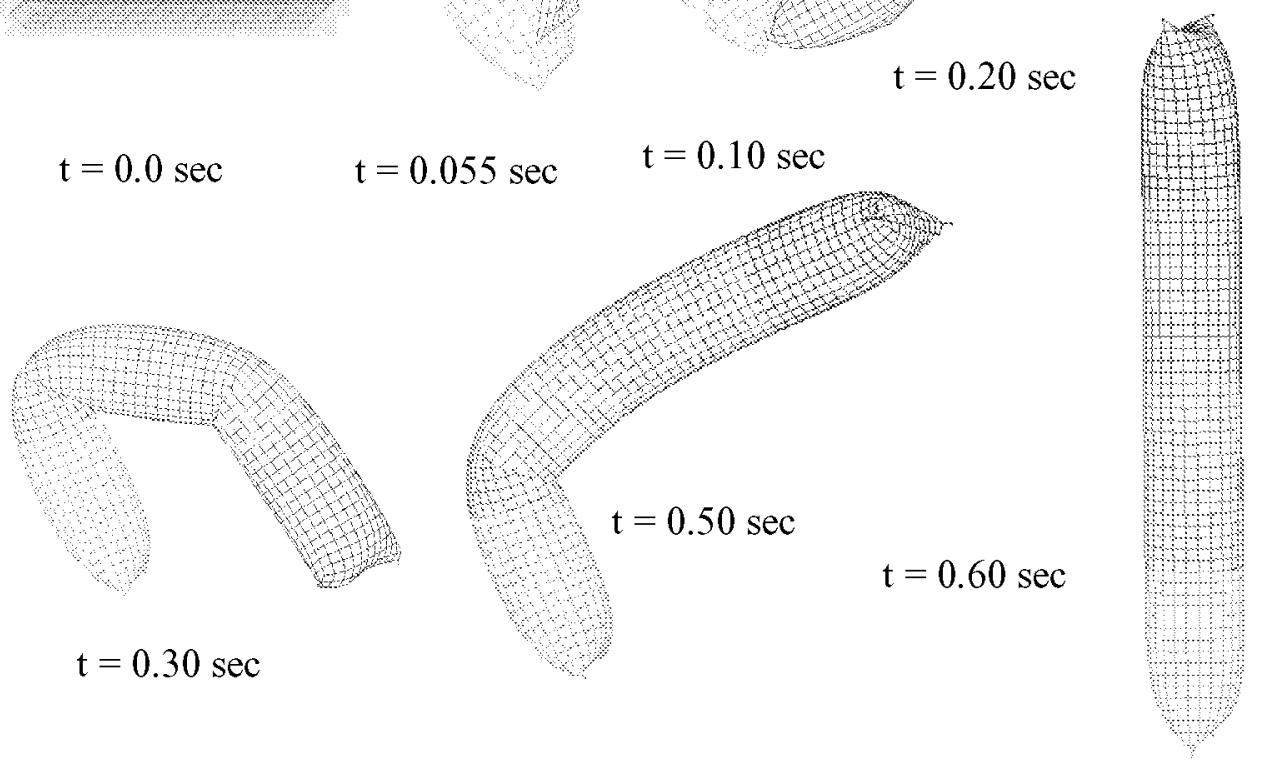

Figure 22. Different deployment stages of the Z-folded tube with end mass and gravity. 


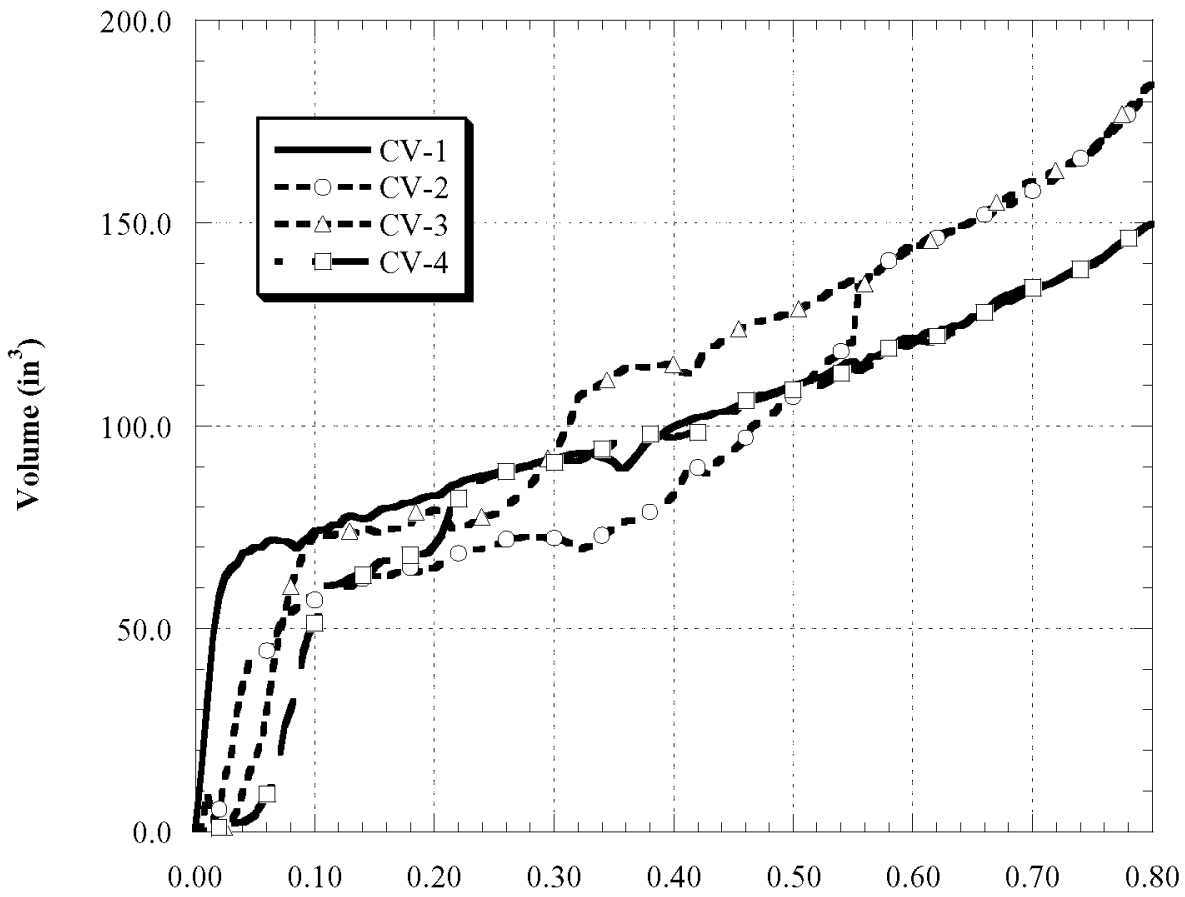

Time (sec)

Figure 23. Volume of each control volume (CV) as a function of time for the gravity case.

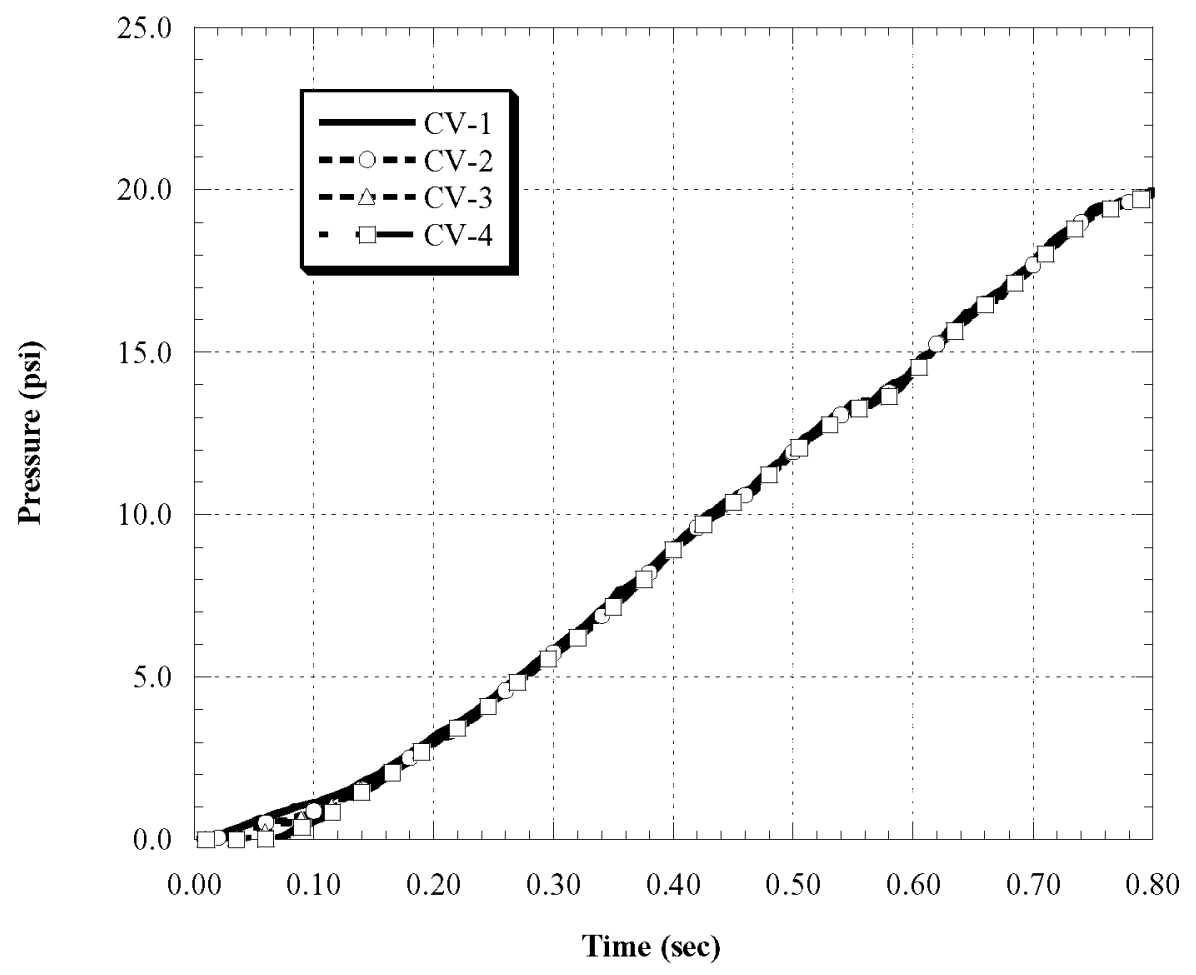

Figure 24. Pressure of each control volume (CV) as a function of time for the gravity case. 